Notre Dame Journal of Formal Logic

Volume 46, Number 4, 2005

\title{
First-Order Modal Logic with an 'Actually' Operator
}

\author{
Yannis Stephanou
}

\begin{abstract}
In this paper the language of first-order modal logic is enriched with an operator @ ('actually') such that, in any model, the evaluation of a formula @ A at a possible world depends on the evaluation of $\mathbf{A}$ at the actual world. The models have world-variable domains. All the logics that are discussed extend the classical predicate calculus, with or without identity, and conform to the philosophical principle known as serious actualism. The basic logic relies on the system $\mathbf{K}$, whereas others correspond to various properties that the actual world may have. All the logics are axiomatized.
\end{abstract}

\section{Introduction}

We can envisage an operator @ which always, even if it occurs within the scope of other modal operators, introduces information about what is in fact the case. Equipped with @, we can formalize sentences such as

It could have been that every singer who is in fact rich was poor and

There could have been a table that does not actually exist.

The former sentence can be rendered as $\diamond(\forall x)[(S x \wedge @ R x) \rightarrow P x]$, while the latter is paraphrasable as $\diamond(\exists x)[T x \wedge \neg @(\exists y)(x=y)]$. In this paper, @ will be added to the usual language of first-order modal logic.

What makes@ interesting is that the concept of actuality should be central in modal logic. Necessity, actuality, and possibility are notions that are closely linked together and equally central to our thinking about how things could be. So, to the extent that modal logic aims to articulate the logical principles to which such thinking ought to conform, one would expect it to focus on all three notions. But, in fact, necessity and possibility were given pride of place, whereas actuality was relatively

Received July 21, 2004; accepted October 19, 2004; printed December 8, 2005

2000 Mathematics Subject Classification: Primary, 03B45

Keywords: 'actually' operators, first-order modal logic

(C)2005 University of Notre Dame 
neglected. The reason for that neglect must be that apparently we do not need the notion of actuality in order to say how things actually are: we can just say 'The earth moves', without adding words like 'in fact'. It is true that we do not need actuality if we are not speaking in the scope of a modal operator. But if within the scope of a necessity or possibility operator we want to speak about how things actually are, then we must in some way or other use the concept of actuality. ${ }^{1}$

Operators such as @ have been studied in propositional modal logic (e.g., Crossley and Humberstone [2], Gregory [3], and Blackburn and Marx [1]). There has also been a study of @ in first-order modal logic based on S5 (Hodes [4]). The treatment of @ here will be much more general; the basic axiomatization we shall discuss is based on the system $\mathbf{K}$ of standard modal logic.

Our models will involve possible worlds. In each model, one world is characterized as actual. When a formula is evaluated in a model, every occurrence of @ turns the evaluation to the actual world of the model. The domains of quantification will generally vary from world to world. So, in most of the axiomatizations to be discussed, neither the Barcan Formula, $(\forall \mathbf{x}) \square \mathbf{A} \rightarrow \square(\forall \mathbf{x}) \mathbf{A}$, nor its converse, $\square(\forall \mathbf{x}) \mathbf{A} \rightarrow(\forall \mathbf{x}) \square \mathbf{A}$, is a theorem schema. The language will contain individual constants that are treated as rigid designators.

The axiomatizations will all extend the classical predicate calculus: half of them extend a formulation of the calculus without identity while the other half extend a formulation with identity. So this paper is part of the effort to develop first-order modal logic as an extension of classical first-order logic. The language will have no primitive existence predicate, not because of any philosophical objections to treating existence as a property, but because no primitive existence predicate appears in standard formulations of classical first-order logic.

Within modal contexts, all axiomatic systems allow existential generalization from atomic formulas; correspondingly, the models have local predicates (i.e., the extension of a predicate letter at a world is confined to objects drawn from the domain of that world). This feature of the systems is a formal analogue of the metaphysical view known as serious actualism: for every entity $e$ and every property $P$ or relation $R$, it could not have been that $e$ had $P$, or bore $R$, but did not exist. So the paper accords with that very plausible metaphysical view. Serious actualism is part of Plantinga's philosophy of modality; see, for example, Plantinga [6], pp. 31323 and 344-47. Also, in the absence of an existence predicate, local predicates are helpful technically.

This paper extends the results of Stephanou [7] to modal logic with @. So, many, though by no means all, of the metatheorems that are proved below have parallels in that paper. The proofs provided here are generally different from those found there. Different proofs are more interesting, but when two parallel results are proved differently in the respective papers, each one can be proved in the other way. ${ }^{2}$ At any rate, some proofs for the present paper are similar to proofs in the other paper and have been replaced with references to the latter.

Of the two sections that follow, the one contains the claims to be proved, and the other contains the proofs.

\section{Systems and Results}

Our object language will be $\mathcal{L}$, which has these symbols: 


$$
\begin{array}{lll}
x_{1}, x_{2}, \ldots & & \text { (variables) } \\
a_{1}, a_{2}, \ldots & & \text { (individual constants) } \\
F_{1}^{1}, F_{2}^{1}, \ldots, \quad F_{1}^{2}, F_{2}^{2}, \ldots, & \ldots & \text { (predicate letters) } \\
\rightarrow, \quad \neg, \quad \square, \quad @, \quad \forall, \quad[, \quad], \quad(, \quad) . &
\end{array}
$$

When the variables or the predicate letters of degree 1 are arranged in order of increasing subscripts, their order will be called alphabetical. As usual, the predicate letters of degree $i$ are those whose superscript is $i$.

The letters A, B, C, D, and $\mathbf{E}$ (with or without a prime or subscript) will be used as metalinguistic variables ranging over the well-formed formulas (wffs) of $\mathcal{L} ; \mathbf{x}, \mathbf{y}$, $\mathbf{z}$, and $\mathbf{v}$ range over the variables of $\mathcal{L}$, and $\mathbf{a}$ over the individual constants; $\mathbf{b}, \mathbf{c}$, and $\mathbf{d}$ range over both the variables and the individual constants; $\mathbf{F}$ and $\mathbf{G}$ range over the predicate letters. The letters $m, n$, and $l$ will be variables ranging over the natural numbers, while $i, j$, and $k$ range over the positive integers.

A series of symbols is an atomic wff just in case it has the form $F_{j}^{i} \mathbf{b}_{1} \ldots \mathbf{b}_{i}$. For any $\mathbf{A}, \mathbf{B}$, and $\mathbf{x}$, the formulas $[\mathbf{A} \rightarrow \mathbf{B}], \neg \mathbf{A}, \square \mathbf{A}$, @A A and $(\forall \mathbf{x}) \mathbf{A}$ are well formed. Nothing else is well formed. Other connectives and the existential quantifier are introduced by standard abbreviatory definitions. ${ }^{3}$ Standard rules determine when brackets may be omitted. ${ }^{4}$

As usual, $\mathbf{b}$ is free for $\mathbf{x}$ in $\mathbf{A}$ if and only if in $\mathbf{A}$ no free occurrence of $\mathbf{x}$ is in the scope of an occurrence of $(\forall \mathbf{b})$. $\dot{\mathbf{S}}_{\mathbf{b}}^{\mathbf{x}} \mathbf{A}$ is the wff that results from $\mathbf{A}$ when we substitute $\mathbf{b}$ for every free occurrence of $\mathbf{x}$.

A structure is a quintuple $\left\langle W, R, D, Q, w^{*}\right\rangle . W$ and $D$ are nonempty sets. $R$ and $Q$ are relations: $R$ (the accessibility relation) is a subset of $W \times W$, and $Q$ is a subset of $W \times D$. The members of $W$ and $D$ will be called 'worlds' and 'individuals', respectively. For every $w \in W, \operatorname{dom}(w)$ (the domain of $w$ ) will be the range of the restriction of $Q$ to $\{w\}$. Finally, $w^{*}$ (the actual world) is a member of $W$ such that $\operatorname{dom}\left(w^{*}\right)$ is nonempty. Any such quintuple is a structure.

A model is a sextuple $\left\langle W, R, D, Q, w^{*}, V\right\rangle$, where $\left\langle W, R, D, Q, w^{*}\right\rangle$ is a structure. $V$ is a function such that, for each individual constant $\mathbf{a}, V(\mathbf{a})$ is a member of $\operatorname{dom}\left(w^{*}\right)$ while, for each pair $\left\langle F_{j}^{i}, w\right\rangle$ of a predicate letter and a world belonging to $W, V\left(F_{j}^{i}, w\right)$ is a subset of $(\operatorname{dom}(w))^{i}$. Any such sextuple is a model. We shall say that the model $\left\langle W, R, D, Q, w^{*}, V\right\rangle$ is based on the structure $\left\langle W, R, D, Q, w^{*}\right\rangle$.

Given a model, we can define what it means for a (denumerable) sequence $s$ of individuals to satisfy a wff at a world. We first define the function $s^{*}: s^{*}\left(x_{i}\right)=s_{i}$ and $s^{*}(\mathbf{a})=V(\mathbf{a})$.

1. $s$ satisfies $F_{j}^{i} \mathbf{b}_{1} \ldots \mathbf{b}_{i}$ at $w$ if and only if $\left\langle s^{*}\left(\mathbf{b}_{1}\right), \ldots, s^{*}\left(\mathbf{b}_{i}\right)\right\rangle \in V\left(F_{j}^{i}, w\right)$.

2. $s$ satisfies $\mathbf{A} \rightarrow \mathbf{B}$ at $w$ if and only if either $s$ does not satisfy $\mathbf{A}$ at $w$ or $s$ satisfies $\mathbf{B}$ at $w$.

3. $s$ satisfies $\neg \mathbf{A}$ at $w$ if and only if $s$ does not satisfy $\mathbf{A}$ at $w$.

4. $s$ satisfies $\square \mathbf{A}$ at $w$ if and only if, for every world $w^{\prime}$ such that $w R w^{\prime}, s$ satisfies $\mathbf{A}$ at $w^{\prime}$.

5. $s$ satisfies @ A at $w$ if and only if $s$ satisfies $\mathbf{A}$ at $w^{*}$.

6. $s$ satisfies $\left(\forall x_{i}\right) \mathbf{A}$ at $w$ if and only if every sequence $s^{\prime}$ of individuals which differs from $s$ in at most the $i$ th position and is such that $s_{i}^{\prime} \in \operatorname{dom}(w)$ satisfies A at $w$. 
A wff is true in the model if and only if it is satisfied at $w^{*}$ by every sequence of elements of $\operatorname{dom}\left(w^{*}\right)$.

A wff is valid in a structure $\left\langle W, R, D, Q, w^{*}\right\rangle$ if and only if it is true in every model based on the structure. A set $\Lambda$ of wffs is satisfiable in $\left\langle W, R, D, Q, w^{*}\right\rangle$ if and only if, in some model based on the structure, there is a sequence of elements of $\operatorname{dom}\left(w^{*}\right)$ which satisfies all members of $\Lambda$ at $w^{*}$. A wff $\mathbf{A}$ is a consequence of $\Lambda$ in $\left\langle W, R, D, Q, w^{*}\right\rangle$ if and only if, in every model based on the structure, every sequence of elements of $\operatorname{dom}\left(w^{*}\right)$ that satisfies all members of $\Lambda$ at $w^{*}$ satisfies $\mathbf{A}$, too, at $w^{*}$. The notion of consequence generalizes that of validity in the usual way: a wff is valid in a structure just in case it is a consequence of the empty set in that structure.

The basic axiomatic system we shall discuss is @ K. Apart from axiom schemata and primitive rules of inference, @ $\mathrm{K}$ also has a rule that imposes a restriction on how a proof may develop after certain axiom schemata have been invoked. The axiomatic system is the following.

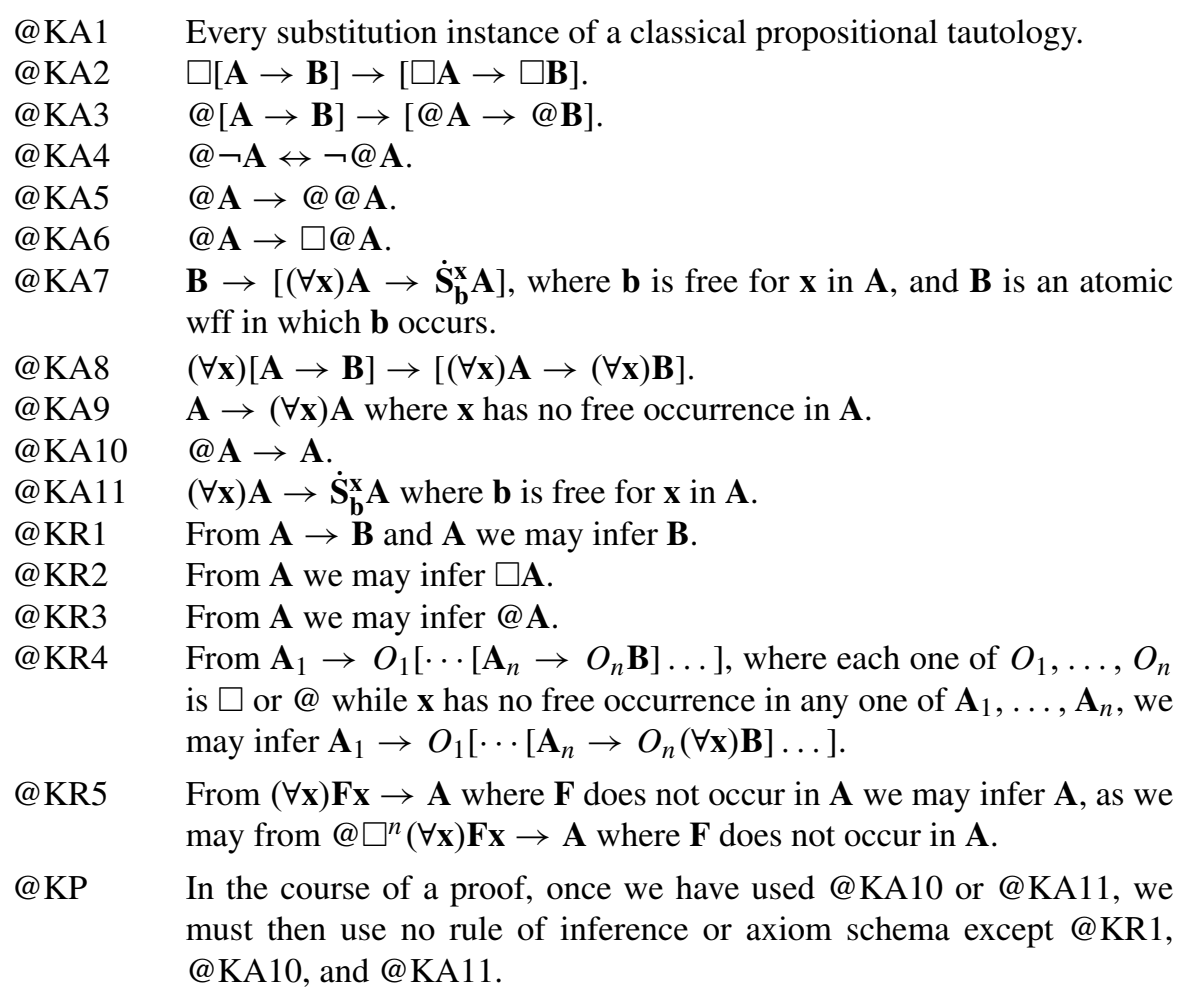

Note that @KR4 subsumes the rule of universal generalization, since every inference from a wff $\mathbf{B}$ to a wff $(\forall \mathbf{x}) \mathbf{B}$ is an instance of @KR4 in which $n=0$. The symbol $\square^{n}$ means that zero, one, or more boxes may occur in its position.

Resorting to a rule such as @ KR5 is necessary because our language has no primitive existence predicate. If we had one, $E$, we could employ the axiom schema $(\forall \mathbf{x}) E \mathbf{x}$. @ KR5 will play a role in our proofs similar to the one that would be played 
by $(\forall \mathbf{x}) E \mathbf{x}$. Indeed, the intuitive idea behind @KR5 also involves the notion of existence. If a wff $(\forall \mathbf{x}) \mathbf{F x} \rightarrow \mathbf{A}$ or $@ \square^{n}(\forall \mathbf{x}) \mathbf{F x} \rightarrow \mathbf{A}$ has been established, then it holds however we may read its schematic letters (that is, the predicate letters and the individual constants). In particular, it holds if we construe $\mathbf{F}$ as meaning 'exists'. But then $(\forall \mathbf{x}) \mathbf{F x}$ ('Every entity exists') not only is true but remains so when we prefix it with as many occurrences of @ and $\square$ as we like. So A also holds however we may read its schematic letters (which do not include $\mathbf{F}$ ).

@KP helps to determine what counts as a proof in @ $\mathrm{K}$ (or in any other of the axiomatic systems we shall discuss). A proof here is a $k$-tuple of ordered pairs $\langle\mathbf{A}, \mathbf{s}\rangle$ where $\mathbf{s}$ is an axiom schema or primitive rule of inference. Such a $k$-tuple counts as a proof (of the wff in the last pair) if and only if each $\left\langle\mathbf{A}_{i}, \mathbf{s}_{i}\right\rangle(1 \leq i \leq k)$ satisfies two conditions. The one condition is to be expected: either $\mathbf{s}_{i}$ is an axiom schema and $\mathbf{A}_{i}$ is an instance of it, or $\mathbf{s}_{i}$ is a rule and among $\mathbf{A}_{1}, \ldots, \mathbf{A}_{i-1}$ there are one or more wffs from which $\mathbf{A}_{i}$ can be immediately inferred in accordance with that rule. The other condition is specified by @KP: if $\mathbf{s}_{i}$ is @KA10 or @KA11, then every $\mathbf{s}_{j}$ such that $i<j \leq k$ is@ $@ \mathrm{KR} 1$, @ KA10, or @ KA11.

If we use @KA10 or @KA11 in a proof in @K, the proof consists of two parts. The first part, which may be empty and ends just before the first wff for which we use one of those schemata, relies at most on @KA1-9 and @KR1-5. The second part, which begins with the first wff for which we use @KA10 or @KA11, relies at most on @KR1, @KA10-11, and everything that has been proved in the first part. The first wff for which we use @ KA10 or @ KA11 is the wff in the first pair $\langle\mathbf{A}, \mathbf{s}\rangle$ such that $\mathbf{s}$ is one of them. When we extend @ K by adding further axiom schemata, @KP will still be in force, so the additional axiom schemata must not be used in a proof after @KA10 or @KA11 has been invoked.

As will be shown, the theorems of @K are the wffs that are valid in every structure; in other words, @ $\mathrm{K}$ is sound and weakly complete regarding validity in all structures. Moreover, satisfiability in a structure is compact: it is the case for any set $\Lambda$ of wffs that if, for each finite subset of $\Lambda$, there is a structure in which that subset is satisfiable, then $\Lambda$ itself is satisfiable in a structure. And @ $\mathrm{K}$ is strongly complete regarding consequence in all structures: it is the case for any $\mathbf{A}$ and any set $\Lambda$ of wffs that if $\mathbf{A}$ is a consequence of $\Lambda$ in every structure, then for some subset $\left\{\mathbf{B}_{1}, \ldots, \mathbf{B}_{n}\right\}$ of $\Lambda(n \geq 0) \vdash @ \mathrm{~K} \mathbf{B}_{1} \wedge \cdots \wedge \mathbf{B}_{n} \rightarrow \mathbf{A}$.

If an axiomatic system for $\mathcal{L}$ is intended as an extension of classical first-order logic, all wffs of the form $(\forall \mathbf{x}) \mathbf{A} \rightarrow \dot{\mathbf{S}}_{\mathbf{b}}^{\mathbf{x}} \mathbf{A}$, where $\mathbf{b}$ is free for $\mathbf{x}$ in $\mathbf{A}$, should be theorems. However, applying the rule of necessitation, @KR2, to a wff of that form will normally lead to a formula that is not a correct principle. For example, $\square\left[\left(\forall x_{1}\right) F_{1}^{1} x_{1} \rightarrow F_{1}^{1} a_{1}\right]$ seems to be incorrect in the sense that, when we replace the predicate letter and the individual constant with a real predicate and a real name, respectively, the result is not always a truth. ${ }^{\prime} \square\left[\left(\forall x_{1}\right)\left(x_{1}\right.\right.$ is a material object) $\rightarrow$ (the Taj Mahal is a material object)]' is not true. It could have been that every entity was a material object but the Taj Mahal did not exist at all and so, a fortiori, was not a material object. Again, if an axiomatic system for $\mathcal{L}$ is intended to capture the logic of the operator@, all wffs of the form@A $\rightarrow$ A should be theorems. Since @ was defined as introducing information about what is in fact the case, it is part of the logic of this operator that all statements @ $p \rightarrow p$ are true. However, applying the rule of necessitation to a wff of the form @ $\mathbf{A} \rightarrow \mathbf{A}$ will normally lead to a formula that is not a correct principle. For instance, $\square\left[@ F_{1}^{1} a_{1} \rightarrow F_{1}^{1} a_{1}\right]$ fails upon 
many replacements of the predicate letter and the individual constant. ' $\square @$ (Plato is a philosopher)' is true, but ' $\square$ (Plato is a philosopher)' is not, for Plato could have led a different life.

QKP allows us to count all wffs of the form $(\forall \mathbf{x}) \mathbf{A} \rightarrow \dot{\mathbf{S}}_{\mathbf{b}}^{\mathbf{x}} \mathbf{A}$ (where $\mathbf{b}$ is free for $\mathbf{x}$ in $\mathbf{A}$ ), as well as those of the form @ $\mathbf{A} \rightarrow \mathbf{A}$, as theorems while generally avoiding their necessitations. Correspondingly, the definition of truth in a model has been so engineered that all wffs of those two forms turn out to be true in all models while generally their necessitations do not. The main factor is that truth in a model was defined as satisfaction at the actual world and not at every world. This choice not only allows a wff to be true in every model without its necessitation sharing this feature but also, given the definition of satisfaction, suffices to ensure that the wffs of the form @ $\mathbf{A} \rightarrow \mathbf{A}$ are true in all models. On the other hand, to ensure that those of the form $(\forall \mathbf{x}) \mathbf{A} \rightarrow \dot{\mathbf{S}}_{\mathbf{b}}^{\mathbf{x}} \mathbf{A}$ are true in all models, we also had to confine variables and individual constants to values drawn from the domain of the actual world. So, an appropriate clause about individual constants was inserted in the definition of a model, and truth in a model was defined in terms of satisfaction by sequences that take values only from $\operatorname{dom}\left(w^{*}\right)$.

It is interesting to see how we can axiomatize various conditions that a structure may meet. There are conditions that concern the accessibility relation, and there are others that concern the domains of the worlds. Here are some conditions of the former kind.

1. For every world $w, w^{*} R w$.

2. For every $w, w R w^{*}$.

3. For any worlds $w$ and $w^{\prime}$, if $w^{*} R w^{\prime}$ then $w R w^{\prime}$.

4. For any $w$ and $w^{\prime}$, if $w R w^{\prime}$ then $w^{*} R w^{\prime}$.

5. For any $w$ and $w^{\prime}, w R w^{\prime}$.

And here are some of the latter kind.

6. For every world $w, \operatorname{dom}(w)$ and $\operatorname{dom}\left(w^{*}\right)$ are disjoint unless $w$ is $w^{*}$.

7. For every $w, \operatorname{dom}(w)$ and $\operatorname{dom}\left(w^{*}\right)$ have a common element.

8. For every $w, \operatorname{dom}(w) \subseteq \operatorname{dom}\left(w^{*}\right)$.

9. For every $w, \operatorname{dom}\left(w^{*}\right) \subseteq \operatorname{dom}(w)$.

10. For any worlds $w$ and $w^{\prime}, \operatorname{dom}(w)$ and $\operatorname{dom}\left(w^{\prime}\right)$ are disjoint unless $w$ is $w^{\prime}$.

11. For any $w$ and $w^{\prime}, \operatorname{dom}(w)=\operatorname{dom}\left(w^{\prime}\right)$.

Finally, this is an example of a condition that concerns both the accessibility relation and the domains.

12. For every world $w$, if $w^{*} R w$ then $\operatorname{dom}\left(w^{*}\right)$ and $\operatorname{dom}(w)$ are disjoint.

(1) - (12) are conditions that either involve the actual world or can be captured by axiom schemata that involve the symbol @.

We shall employ the following axiom schemata.

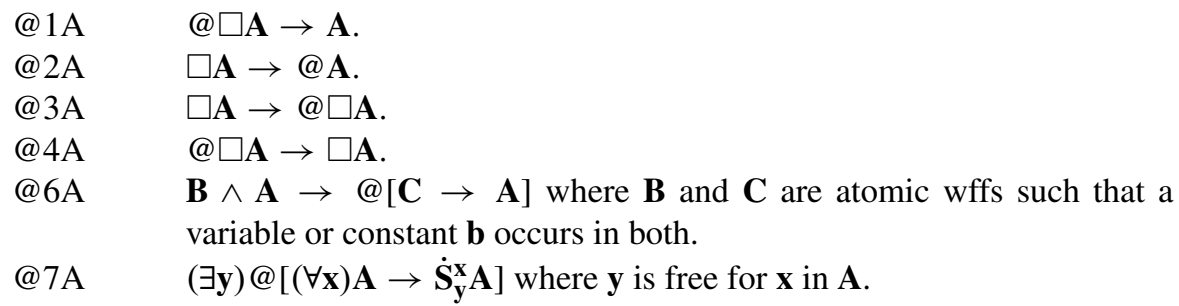


@ 8A $\mathbf{B} \rightarrow @\left[(\forall \mathbf{x}) \mathbf{A} \rightarrow \dot{\mathbf{S}}_{\mathbf{b}}^{\mathbf{x}} \mathbf{A}\right]$, where $\mathbf{b}$ is free for $\mathbf{x}$ in $\mathbf{A}$, and $\mathbf{B}$ is an atomic wff in which $\mathbf{b}$ occurs.

$@ 9 A \quad @ \mathbf{B} \rightarrow\left[(\forall \mathbf{x}) \mathbf{A} \rightarrow \dot{\mathbf{S}}_{\mathbf{b}}^{\mathbf{x}} \mathbf{A}\right]$, where $\mathbf{b}$ is free for $\mathbf{x}$ in $\mathbf{A}$, and $\mathbf{B}$ is an atomic wff in which $\mathbf{b}$ occurs.

$@ 10 \mathrm{~A} \quad \mathbf{B} \wedge \mathbf{A} \rightarrow @ \square^{n}[\mathbf{C} \rightarrow \mathbf{A}]$ where $\mathbf{B}$ and $\mathbf{C}$ are atomic wffs such that a variable or constant $\mathbf{b}$ occurs in both.

@12A @ $@(\forall \mathbf{x}) \square \neg \mathbf{A}$ where $\mathbf{A}$ is an atomic wff in which $\mathbf{x}$ occurs.

For every $j$ such that $1 \leq j \leq 4$ or $6 \leq j \leq 10$ or $j=12$, the axiomatic system @ $j$ will be @ + + @ $j \mathrm{~A}$. The axiomatic systems @5 and @11 will be @ + + @1A $+@ 3 \mathrm{~A}$ and $@ \mathrm{~K}+@ 8 \mathrm{~A}+@ 9 \mathrm{~A}$, respectively.

It will be shown that, for every $j(1 \leq j \leq 12)$, @ $j$ is sound and weakly complete regarding validity in all structures meeting condition $(j)$. Moreover, satisfiability in a structure meeting $(j)$ is compact, and @ $j$ is strongly complete regarding consequence in all structures meeting $(j)$.

The model-theoretic import of @6A is that if an individual belongs to the domain of a world $w$ and also to the domain of the actual world, $w^{*}$, then any wff $\mathbf{A}$ that is satisfied by a sequence at $w$ is also satisfied by the same sequence at $w^{*}$. To be precise, this is the import of claiming about a model that the instances of @6A are satisfied at every world by every sequence. (If two atomic wffs $\mathbf{B}$ and $\mathbf{C}$ which both contain $\mathbf{b}$ are satisfied by $s$ at two worlds, then the individual $s^{*}(\mathbf{b})$ belongs to both domains.) We indirectly make such a claim, about any models for which our axiomatization will turn out to be appropriate, when we make the instances axioms and allow both the rule of universal generalization and the rule of necessitation to be applied to them; if we only allowed universal generalization, we would only claim that the instances of @6A are satisfied at $w^{*}$ by every sequence of elements of $\operatorname{dom}\left(w^{*}\right)$. Now, condition (6) effectively says that if $\operatorname{dom}(w)$ and $\operatorname{dom}\left(w^{*}\right)$ have a common element, $w$ is $w^{*}$. So it would seem that @6A is too weak to capture (6); it would seem that the axiomatization @6 is not complete regarding validity in all structures meeting (6). For in a model it may be that, for some world $w$, every wff that is satisfied by a sequence at $w$ is also satisfied by the sequence at $w^{*}$ but $w$ and $w^{*}$ are distinct. In fact, however, @6A suffices, as will be shown in our completeness proof. The proof will focus on canonical models, in which if a certain canonical sequence satisfies at $w^{\prime}$ every wff it satisfies at $w$, then $w$ is $w^{\prime}$.

Similarly, the model-theoretic import of @10A is that if an individual belongs to the domain of a world $w$ and also to the domain of a world $w^{\prime}$ that is $n R$-steps away from the actual world, then any wff $\mathbf{A}$ that is satisfied by a sequence at $w$ is also satisfied by the same sequence at $w^{\prime} .5$ Condition (10) effectively says that if $\operatorname{dom}(w)$ and $\operatorname{dom}\left(w^{\prime}\right)$ have a common element, $w$ is $w^{\prime}$. Although the condition refers to every world $w^{\prime}$, and not just every world that is $n R$-steps away from the actual one, @10A suffices to capture (10). In the canonical models involved in the proof, for each world $w^{\prime}$ there will be some $n$ such that $w^{\prime}$ is $n R$-steps away from the actual world.

When we discuss first-order modal logic with identity, we shall use the letter $F_{1}^{2}$ as a sign of identity. [ $\mathbf{b}=\mathbf{c}]$ is defined as $F_{1}^{2} \mathbf{b c}$. The brackets will be omitted unless omitting them would result in unclarity. A model is an identity model if and only if, for every world $w$ and all individuals $d$ and $d^{\prime}$ in $\operatorname{dom}(w),\left\langle d, d^{\prime}\right\rangle \in V\left(F_{1}^{2}, w\right)$ just in case $d$ is $d^{\prime}$. A wff is I-valid in a structure $\left\langle W, R, D, Q, w^{*}\right\rangle$ if and only if it is 
true in every identity model based on the structure. A set $\Lambda$ of wffs is I-satisfiable in $\left\langle W, R, D, Q, w^{*}\right\rangle$ if and only if, in some identity model based on the structure, there is a sequence of elements of $\operatorname{dom}\left(w^{*}\right)$ which satisfies all members of $\Lambda$ at $w^{*}$. And a wff $\mathbf{A}$ is an I-consequence of $\Lambda$ in $\left\langle W, R, D, Q, w^{*}\right\rangle$ if and only if, in every identity model based on the structure, every sequence of elements of $\operatorname{dom}\left(w^{*}\right)$ that satisfies all members of $\Lambda$ at $w^{*}$ satisfies $\mathbf{A}$, too, at $w^{*}$. So a wff is I-valid in a structure just in case it is an I-consequence of the empty set in that structure.

Our basic axiomatization with identity will be @IK, which results from @K by adding these axiom schemata.

$$
\begin{aligned}
& \text { @IKA1 }(\forall \mathbf{x})[\mathbf{x}=\mathbf{x}] \text {. } \\
& \text { @IKA2 } \mathbf{x}=\mathbf{y} \rightarrow[\mathbf{A} \rightarrow \mathbf{B}] \text {, where } \mathbf{A} \text { is an atomic wff in which } \mathbf{x} \text { occurs, and } \mathbf{B} \\
& \text { results from } \mathbf{A} \text { by substituting } \mathbf{y} \text { for an occurrence of } \mathbf{x} \text {. }
\end{aligned}
$$

@IKA3 $\mathbf{x}=\mathbf{y} \rightarrow \square^{n}[\mathbf{A} \rightarrow \mathbf{x}=\mathbf{y}]$ where $\mathbf{A}$ is an atomic wff in which $\mathbf{x}$ occurs or $\mathbf{y}$ occurs.

As we shall see, the theorems of @ IK are the wffs that are I-valid in every structure; that is, @IK is sound and weakly complete regarding I-validity in all structures. Further, I-satisfiability in a structure is compact: it is the case for any set $\Lambda$ of wffs that if, for each finite subset of $\Lambda$, there is a structure in which that subset is Isatisfiable, then $\Lambda$ itself is I-satisfiable in a structure. And @IK is strongly complete regarding I-consequence in all structures: it is the case for any $\mathbf{A}$ and any set $\Lambda$ of wffs that if $\mathbf{A}$ is an I-consequence of $\Lambda$ in every structure, then for some subset $\left\{\mathbf{B}_{1}, \ldots, \mathbf{B}_{n}\right\}$ of $\Lambda(n \geq 0) \vdash @ \mathrm{IK} \mathbf{B}_{1} \wedge \cdots \wedge \mathbf{B}_{n} \rightarrow \mathbf{A}$.

Each instance of the schema @IKA3 is a variant on the principle of the necessity of identity, except for the instances in which $n=0$. (They amount to a similar principle about actuality.) It differs from standard formulations ('If $x$ is $y$ then it is necessarily the case that $x$ is $y^{\prime}$ ) in three respects: the symbol @ occurs in front of the boxes; there may be more than one box; and what follows the boxes has the form $\mathbf{A} \rightarrow \mathbf{x}=\mathbf{y}$, rather than just $\mathbf{x}=\mathbf{y}$. Only the last difference has any philosophical significance. The idea is that, for anything $x$ and anything $y$, it could not have been that $x$ bore the relation of identity to $y$ and yet $x$, that is, $y$, did not exist: if $x$ and $y$ were one and the same thing, then they, or it, would be a thing. This idea is an application of serious actualism to identity. But there are many things that might not have existed. So the necessity of identity should rather take a form such as 'If $x$ is $y$, then it is necessarily the case that, if $x$ or $y$ exists, $x$ is $y^{\prime}$. Every atomic wff $\mathbf{A}$ is treated in this paper as carrying existential commitment with respect to the variables and individual constants it contains. As for the other two differences between @IKA3 and standard formulations of the necessity of identity, they are due to the exigencies of the proof of (3.31) below. It does not seem feasible to deduce @IKA3 from simpler schemata (without resorting to @KA10). If the schema $\mathbf{x}=\mathbf{y} \rightarrow @ \square^{n}[\mathbf{x}=\mathbf{y}]$ were desirable, we could derive it from $\mathbf{x}=\mathbf{y} \rightarrow \square[\mathbf{x}=\mathbf{y}]$ and $\mathbf{x}=\mathbf{y} \rightarrow @[\mathbf{x}=\mathbf{y}]$. But we cannot derive $\mathbf{x}=\mathbf{y} \rightarrow \square^{n}[\mathbf{A} \rightarrow \mathbf{x}=\mathbf{y}]$ from $\mathbf{x}=\mathbf{y} \rightarrow \square[\mathbf{A} \rightarrow \mathbf{x}=\mathbf{y}]$ and $\mathbf{x}=\mathbf{y} \rightarrow @[\mathbf{A} \rightarrow \mathbf{x}=\mathbf{y}]$

For every $j$ such that $1 \leq j \leq 4$ or $6 \leq j \leq 10$ or $j=12$, the axiomatization @I $j$ will be@IK + @jA. The axiomatizations@I5 and @I11 will be@IK + $@ 1 \mathrm{~A}+@ 3 \mathrm{~A}$ and $@ \mathrm{IK}+@ 8 \mathrm{~A}+@ 9 \mathrm{~A}$, respectively. We shall see that, for every $j(1 \leq j \leq 12), @ \mathrm{I} j$ is sound and weakly complete regarding I-validity in all structures meeting condition $(j)$. Moreover, I-satisfiability in a structure meeting $(j)$ 
is compact, and @ $\mathrm{I} j$ is strongly complete regarding I-consequence in all structures meeting $(j)$.

Let $\mathcal{L}^{\prime}$ be the language whose wffs are the wffs of $\mathcal{L}$ in which there is no occurrence of either $\square$ or @. It is the case for any one of the axiomatic systems @ K and $@ j$ that its theorems that are wffs of $\mathscr{L}^{\prime}$ are just the formulas which make up the classical predicate calculus without identity when this calculus is formulated in $\mathscr{L}^{\prime}$. Also, it is the case for any one of the systems @IK and @ $\mathrm{I} j$ that its theorems that are wffs of $\mathcal{L}^{\prime}$ are just the formulas which make up the classical predicate calculus with identity when this calculus is formulated in $\mathcal{L}^{\prime}$ and the sign of identity is $F_{1}^{2}$.

\section{Proofs}

The following four metatheorems will be repeatedly needed later on. $\overline{\mathbf{S}}_{\mathbf{y}}^{\mathbf{x}} \mathbf{A}$ is the wff that results from $\mathbf{A}$ when we substitute $\mathbf{y}$ for every bound occurrence of $\mathbf{x}$.

Metatheorem 3.1 It is the case in every model that for any $\mathbf{A}$, any world $w$, and any sequences $s$ and $s^{\prime}$ of individuals such that, for every variable $x_{i}$ that has a free occurrence in $\mathbf{A}, s_{i}=s_{i}^{\prime}$, s satisfies $\mathbf{A}$ at $w$ if and only if $s^{\prime}$ satisfies $\mathbf{A}$ at $w$.

Metatheorem 3.2 It is the case in every model that for any $\mathbf{A}, \mathbf{x}$, and $\mathbf{b}$ such that $\mathbf{b}$ is free for $\mathbf{x}$ in $\mathbf{A}$, for any world $w$, and for any sequences $s$ and $s^{\prime}$ of individuals such that $s^{*}(\mathbf{b})=s^{\prime *}(\mathbf{x})$ while, for every variable $x_{i}$ that has a free occurrence in $\mathbf{A}$ but is other than $\mathbf{x}, s_{i}=s_{i}^{\prime}, s^{\prime}$ satisfies $\mathbf{A}$ at $w$ if and only if s satisfies $\dot{\mathbf{S}}_{\mathbf{b}}^{\mathbf{x}} \mathbf{A}$ at $w$.

Metatheorem 3.3 It is the case in every model that for any $\mathbf{A}$, any $\mathbf{x}$, any $\mathbf{y}$ that does not occur in $\mathbf{A}$, any world $w$, and any sequence s of individuals, $s$ satisfies $\mathbf{A}$ at $w$ if and only if s satisfies $\overline{\mathbf{S}}_{\mathbf{y}}^{\mathbf{x}} \mathbf{A}$ at $w$.

Metatheorem 3.4 For any predicate letter $\mathbf{F}$, for any models $M=\langle W, R, D, Q$, $\left.w^{*}, V\right\rangle$ and $M^{\prime}=\left\langle W, R, D, Q, w^{*}, V^{\prime}\right\rangle$ where $V$ and $V^{\prime}$ differ in at most what they assign to $\mathbf{F}$, for any $\mathbf{A}$ in which $\mathbf{F}$ does not occur, for any world $w \in W$, and for any sequence s of members of $D, s$ satisfies $\mathbf{A}$ at $w$ in $M$ if and only if $s$ satisfies $\mathbf{A}$ at $w$ in $M^{\prime}$.

(3.1) - (3.4) can be proved by induction on the number of occurrences of $\rightarrow, \neg, \square$, $@$, and $\forall$ in A. The proof of (3.2) presupposes (3.1), and the proof of (3.3) presupposes (3.2).

A wff is $W$-true in a model if and only if it is satisfied at every world by every sequence of individuals. A wff, such as @ $F_{1}^{1} a_{1} \rightarrow F_{1}^{1} a_{1}$, may be valid in a structure without being $\mathrm{W}$-true in every model based on the structure. The next three metatheorems about W-truth are needed for demonstrating (3.8), which will be pivotal for soundness.

Metatheorem 3.5 If $\mathbf{A}_{1} \rightarrow O_{1}\left[\cdots\left[\mathbf{A}_{n} \rightarrow O_{n} \mathbf{B}\right] \ldots\right]$, where each one of $O_{1}, \ldots, O_{n}$ is $\square$ or @, is $W$-true in a model, then $\mathbf{A}_{1} \rightarrow O_{1}\left[\cdots\left[\mathbf{A}_{n} \rightarrow\right.\right.$ $\left.\left.O_{n}\left(\forall x_{i}\right) \mathbf{B}\right] \ldots\right]$, where $x_{i}$ has no free occurrence in any one of $\mathbf{A}_{1}, \ldots, \mathbf{A}_{n}$, is also W-true in that model.

Proof Suppose that, in the given model and for some sequence $s$ of individuals and some world $w, s$ does not satisfy $\mathbf{A}_{1} \rightarrow O_{1}\left[\cdots\left[\mathbf{A}_{n} \rightarrow O_{n}\left(\forall x_{i}\right) \mathbf{B}\right] \ldots\right]$ at $w$. Then there is an $(n+1)$-tuple $\left\langle w_{0}, \ldots, w_{n}\right\rangle$ of worlds such that $w_{0}$ is $w$; if $O_{j}(1 \leq j \leq n)$ is $\square$ then $w_{j}$ is a world such that $w_{j-1} R w_{j}$, but if $O_{j}$ is @ then $w_{j}$ is $w^{*}$; for every 
$j$ (again $1 \leq j \leq n) s$ satisfies $\mathbf{A}_{j}$ at $w_{j-1}$; and finally $s$ does not satisfy $\left(\forall x_{i}\right) \mathbf{B}$ at $w_{n}$. So there is a sequence $s^{\prime}$ of individuals which differs from $s$ in at most the $i$ th position and does not satisfy $\mathbf{B}$ at $w_{n}$. However, by (3.1), it is the case for every $j$ that $s^{\prime}$ satisfies $\mathbf{A}_{j}$ at $w_{j-1}$. Hence, $s^{\prime}$ does not satisfy $\mathbf{A}_{1} \rightarrow O_{1}\left[\cdots\left[\mathbf{A}_{n} \rightarrow O_{n} \mathbf{B}\right] \ldots\right]$ at $w$.

Metatheorem 3.6 If, for any structure, @ $\square^{n}(\forall \mathbf{x}) \mathbf{F x} \rightarrow \mathbf{A}$, where $\mathbf{F}$ does not occur in $\mathbf{A}$, is W-true in every model based on that structure, then $\mathbf{A}$ is also W-true in every model based on that structure.

Proof Suppose that, in some model $\left\langle W, R, D, Q, w^{*}, V\right\rangle$ based on the given structure, there are a sequence $s$ of individuals and a world $w$ such that $s$ does not satisfy A at $w$. Consider the model $\left\langle W, R, D, Q, w^{*}, V^{\prime}\right\rangle$ where, for every world $w^{\prime}$ that is $n R$-steps away from $w^{*}, V^{\prime}\left(\mathbf{F}, w^{\prime}\right)=\operatorname{dom}\left(w^{\prime}\right)$ but otherwise $V^{\prime}$ does not differ from $V$. In this model, $s$ satisfies @ $\square^{n}(\forall \mathbf{x}) \mathbf{F x}$ at $w$ but, by (3.4), does not satisfy $\mathbf{A}$ at $w$. So @ $\square^{n}(\forall \mathbf{x}) \mathbf{F x} \rightarrow \mathbf{A}$ is not W-true in this model, which is based on the given structure.

Metatheorem 3.7 If, for any structure, $(\forall \mathbf{x}) \mathbf{F} \mathbf{x} \rightarrow \mathbf{A}$, where $\mathbf{F}$ does not occur in $\mathbf{A}$, is W-true in every model based on that structure, then $\mathbf{A}$ is also W-true in every model based on that structure.

Proof Similar to (3.6).

For any set $\Lambda$ of axiom schemata, $@ K+\Lambda$ will be the axiomatic system that results from @K by adding those schemata.

Metatheorem 3.8 If, for any structure, $\Lambda$ is a set of axiom schemata whose instances are $W$-true in every model based on that structure, the theorems of $@ \mathrm{~K}+\Lambda$ are valid in the structure.

Proof A proof in $@ \mathrm{~K}+\Lambda$ divides into two parts, although it may be that one of them is empty: the first part relies at most on @KA1-9, $\Lambda$, and @KR1-5 while the second part relies at most on @KA10-11, @KR1, and everything that has been proved in the first part. Now, the instances of @ KA1-9 are W-true in every model based on the given structure, since they are W-true in all models. (To establish that, use (3.2) in the case of @KA7 and (3.1) in the case of @KA9.) It can easily be shown that for any model the rules @KR1-3 preserve W-truth in that model. By (3.5), for any model the rule @ KR4, too, preserves W-truth in that model. And, by (3.6) and (3.7), for any structure the rule @ KR5 preserves the property of being Wtrue in every model based on that structure. Therefore, everything proved in the first part of the proof in @ $\mathrm{K}+\Lambda$ is W-true in every model based on the given structure, and so it is valid in the structure. Moreover, the instances of @KA10-11 are valid in the given structure (since they are valid in all structures, as can be shown with the help of Metatheorem 3.2) and @KR1 preserves validity in it.

\section{Metatheorem 3.9 If $\vdash @ \mathrm{~K} \mathbf{A}, \mathbf{A}$ is valid in every structure.}

(3.9) is a corollary of (3.8).

In order to demonstrate the completeness of the axiomatizations introduced in Section 2, we must prove some theorems in them. If $\mathbf{a}_{1}, \ldots, \mathbf{a}_{n}$ are distinct individual constants, $\mathbf{S}_{\mathbf{y}_{1}, \ldots, \mathbf{y}_{n}}^{\mathbf{a}_{1}, \ldots, \mathbf{a}_{n}} \mathbf{A}$ is the wff that results from $\mathbf{A}$ when we replace $\mathbf{a}_{1}, \ldots, \mathbf{a}_{n}$ 
with the variables $\mathbf{y}_{1}, \ldots, \mathbf{y}_{n}$, respectively. Let $S$ be an arbitrary axiomatic system which differs from @ $\mathrm{K}$ in at most having additional axiom schemata such that if $\mathbf{A}$ is an instance of one of those schemata, $\mathbf{a}_{1}, \ldots, \mathbf{a}_{n}$ are distinct, and $\mathbf{y}_{1}, \ldots, \mathbf{y}_{n}$ do not occur in $\mathbf{A}$, then $\mathbf{S}_{\mathbf{y}_{1}, \ldots, \mathbf{y}_{n}}^{\mathbf{a}_{1}, \ldots, \mathbf{a}_{n}} \mathbf{A}$ is also an instance of the relevant schema. This restriction on additional axiom schemata is needed for the proof of (3.11) below; all the schemata named in Section 2 comply with it. The notation $\vdash_{S} \mathbf{A}$, of course, means that $\mathbf{A}$ is a theorem of $S$, but the notation $\vdash_{S}^{*} \mathbf{A}$ will mean that $\mathbf{A}$ can be proved in $S$ without using @KA10 or @ KA11; in other words, there is a proof of $\mathbf{A}$ which relies at most on@KA1-9, @KR1-5, and any axiom schemata that $S$ has in addition to those of @K. When the name of a theorem schema ends with a star (e.g., $\left.S \mathrm{~T} 1^{*}\right)$, the star will indicate that neither@KA10 nor @KA11 has been used in the proof.

When we have proved $\mathbf{A}$ and are sketching a proof of a further theorem, it frequently happens that, at some point in the sketch, we invoke $\mathbf{A}$; if we do so in the context of the axiomatizations discussed in this paper, we should make sure that inserting the proof of $\mathbf{A}$ at that point will not lead to a violation of QKP. Easy or standard proofs will normally be omitted. For proofs of $S \mathrm{~T} 10$ and $S \mathrm{~T} 11^{*}$, see [7], p. 203. For a proof of $S \mathrm{~T} 14^{*}$, see [7], p. 204.

$S \mathrm{~T} 1^{*} \quad \square \mathbf{A} \wedge \square \mathbf{B} \rightarrow \square[\mathbf{A} \wedge \mathbf{B}]$.

$S \mathrm{~T} 2 * \quad[@ \mathbf{A} \rightarrow @ \mathbf{B}] \rightarrow @[\mathbf{A} \rightarrow \mathbf{B}]$.

Proof We can first prove @ $\neg \mathbf{A} \rightarrow @[\mathbf{A} \rightarrow \mathbf{B}]$ : begin with $\neg \mathbf{A} \rightarrow[\mathbf{A} \rightarrow \mathbf{B}]$, prefix $@$, and distribute it. Thus, by @KA4, $\neg @ \mathbf{A} \rightarrow @[\mathbf{A} \rightarrow \mathbf{B}]$. We can similarly prove $@ \mathbf{B} \rightarrow @[\mathbf{A} \rightarrow \mathbf{B}]$.

ST3*@@@ $\rightarrow$ @A.

Proof By@KA4 we have $\neg @ \mathbf{A} \rightarrow$ @ $\neg \mathbf{A}$ and $@ \neg @ \mathbf{A} \rightarrow \neg @ @ \mathbf{A} . \quad B y$ $@ \mathrm{KA5}$ we have @ $\neg \mathbf{A} \rightarrow @ @ \neg \mathbf{A}$. By @KA4, @KR3, and @KA3 we have @@ $\neg \mathbf{A} \rightarrow @ \neg @ \mathbf{A}$. Then use @KA1.

ST4* $\quad \neg @ \neg \mathbf{A} \rightarrow @ \mathbf{A}$.

$S \mathrm{~T} 5^{*} \quad @[\mathbf{A} \leftrightarrow \mathbf{B}] \rightarrow[\mathrm{A} \mathbf{A} \leftrightarrow @ \mathbf{B}]$.

ST6* $\quad \neg[@ \mathbf{A} \wedge @ \neg \mathbf{A}]$.

$S T 7^{*} \quad @[\mathbf{A} \wedge \mathbf{B}] \leftrightarrow @ \mathbf{A} \wedge @ \mathbf{B}$.

$S T 8^{*} @ \square[\mathbf{A} \rightarrow \mathbf{B}] \rightarrow[@ \square \mathbf{A} \rightarrow @ \square \mathbf{B}]$.

$S \mathrm{~T} 9^{*} \quad(\forall \mathbf{x})[\mathbf{A} \rightarrow \mathbf{B}] \rightarrow[\mathbf{A} \rightarrow(\forall \mathbf{x}) \mathbf{B}]$ where $\mathbf{x}$ has no free occurrence in $\mathbf{A}$.

$S T 10 \quad(\forall \mathbf{x})\left[(\forall \mathbf{y}) \mathbf{A} \rightarrow \dot{\mathbf{S}}_{\mathbf{b}}^{\mathbf{y}} \mathbf{A}\right]$ where $\mathbf{b}$ is free for $\mathbf{y}$ in $\mathbf{A}$.

SR6 If $\mathbf{B}$ results from $\mathbf{A}$ by substituting $\mathbf{D}$ for an occurrence of $\mathbf{C}$ and $\vdash_{S}^{*} \mathbf{C} \leftrightarrow \mathbf{D}$, then $\vdash_{S}^{*} \mathbf{A} \leftrightarrow \mathbf{B}$.

For a proof of this rule see [7], p. 203; the only difference is that here we should also use @KR3 and $S T 5^{*}$ in the proof. SR6 can be deployed as a derived rule of inference because the way in which it is established provides us with an effective method for getting from a proof of $\mathbf{C} \leftrightarrow \mathbf{D}$ in which @KA10-11 are not used to a proof of $\mathbf{A} \leftrightarrow \mathbf{B}$ in which @KA10-11 are not used.

$S$ T11* $\quad(\forall \mathbf{x}) \mathbf{A} \leftrightarrow(\forall \mathbf{y}) \dot{\mathbf{S}}_{\mathbf{y}}^{\mathbf{x}} \mathbf{A}$ where $\mathbf{y}$ is free for $\mathbf{x}$ in $\mathbf{A}$ and has no free occurrence in $\mathbf{A}$. 
$S \mathrm{~T} 12^{*} \quad \mathbf{A} \leftrightarrow \overline{\mathbf{S}}_{\mathbf{y}}^{\mathbf{x}} \mathbf{A}$ where $\mathbf{y}$ does not occur in $\mathbf{A} .^{6}$

Proof $\quad$ By $S R 6$ and $S T 11^{*}$.

$S$ T13* $\quad(\forall \mathbf{x})[\mathbf{A} \wedge \mathbf{B}] \rightarrow(\forall \mathbf{x}) \mathbf{A} \wedge(\forall \mathbf{x}) \mathbf{B}$.

$S$ T14* $\quad(\forall \mathbf{x}) \mathbf{A} \rightarrow \mathbf{A} \vee(\forall \mathbf{y}) \neg[\mathbf{B} \rightarrow \mathbf{B}]$ where $\mathbf{x}$ has no free occurrence in $\mathbf{A}$.

ST15*@@( $\forall \mathbf{x})[\mathbf{A} \rightarrow @ \mathbf{A}]$.

Proof Let $\mathbf{B}$ be a wff in which $\mathbf{x}$ has no free occurrence and which is a substitution instance of a propositional tautology. From @KA5 and $S \mathrm{~T} 2 *$ we get @ $[\mathbf{A} \rightarrow @ \mathbf{A}]$, so $\mathbf{B} \rightarrow @[\mathbf{A} \rightarrow @ \mathbf{A}]$. Hence, by @KR4, B $\rightarrow @(\forall \mathbf{x})[\mathbf{A} \rightarrow @ \mathbf{A}]$.

$S T 16 * @(\forall \mathbf{x})[@ \mathbf{A} \rightarrow \mathbf{A}]$.

Proof Similar to $S \mathrm{~T} 15^{*}$, but using $S \mathrm{~T} 3^{*}$ instead of @KA5.

$S \mathrm{~T} 17^{*} @(\forall \mathbf{x}) \mathbf{A} \rightarrow @(\forall \mathbf{x}) @ \mathbf{A}$.

ST18 $\quad(\forall \mathbf{x})[@ \mathbf{A} \rightarrow \mathbf{A}]$.

SR7 If $\vdash_{S}^{*} \mathbf{A} \rightarrow @ \square \mathbf{B}$ and $\mathbf{x}$ has no free occurrence in $\mathbf{A}$, then $\vdash_{S}^{*} \mathbf{A} \rightarrow$ $@ \square(\forall \mathbf{x}) \mathbf{B}$.

Proof Let $\mathbf{C}$ be a wff in which $\mathbf{x}$ has no free occurrence and which is a substitution instance of a propositional tautology. We can easily show that $\vdash_{S}^{*} @ \square \mathbf{B} \rightarrow$ $@[\mathbf{C} \rightarrow \square \mathbf{B}]$. Thus, since $\vdash_{S}^{*} \mathbf{A} \rightarrow @ \square \mathbf{B}$, we have $\vdash_{S}^{*} \mathbf{A} \rightarrow @[\mathbf{C} \rightarrow \square \mathbf{B}]$. Hence, by @KR4, $\vdash_{S} \mathbf{A} \rightarrow @[\mathbf{C} \rightarrow \square(\forall \mathbf{x}) \mathbf{B}]$. But we can also easily show that $\vdash_{S}^{*} @[\mathbf{C} \rightarrow \square(\forall \mathbf{x}) \mathbf{B}] \rightarrow @ \square(\forall \mathbf{x}) \mathbf{B}$.

SR8 If $\vdash_{S}^{*} \mathbf{A} \rightarrow @ \square\left[\mathbf{C}_{1} \rightarrow \square\left[\cdots\left[\mathbf{C}_{i} \rightarrow \square \mathbf{B}\right] \ldots\right]\right]$ and $\mathbf{x}$ has no free occurrence in any one of $\mathbf{A}, \mathbf{C}_{1}, \ldots, \mathbf{C}_{i}$, then $\vdash_{S}^{*} \mathbf{A} \rightarrow @ \square\left[\mathbf{C}_{1}\right.$ $\left.\rightarrow \square\left[\cdots\left[\mathbf{C}_{i} \rightarrow \square(\forall \mathbf{x}) \mathbf{B}\right] \ldots\right]\right]$.

The proof of $S R 8$ is similar to that of $S \mathrm{R} 7$.

A set $\Lambda$ of wffs is $S$-consistent if and only if there are no wffs $\mathbf{A}_{1}, \ldots, \mathbf{A}_{i} \in \Lambda$ $(i \geq 1)$ such that $\vdash_{S} \neg\left[\mathbf{A}_{1} \wedge \cdots \wedge \mathbf{A}_{i}\right]$. It is $S$-consistent ${ }^{*}$ if and only if there are no wffs $\mathbf{A}_{1}, \ldots, \mathbf{A}_{i} \in \Lambda(i \geq 1)$ such that $\vdash_{S}^{*} \neg\left[\mathbf{A}_{1} \wedge \cdots \wedge \mathbf{A}_{i}\right] . \Lambda$ is maximal if and only if, for each $\mathbf{A}, \mathbf{A} \in \Lambda$ or $\neg \mathbf{A} \in \Lambda$. The following metatheorem is standard.

Metatheorem 3.10 It is the case for any maximal $S$-consistent* ${ }^{*}$ set $\Gamma$ of wffs that if $\vdash_{S}^{*} \mathbf{A}$ then $\mathbf{A} \in \Gamma$, and that if $\Gamma$ contains both $\mathbf{A} \rightarrow \mathbf{B}$ and $\mathbf{A}$ it also contains $\mathbf{B}$.

$\Lambda$ is an actuality set if and only if every one of its members begins with @. It is finite* just in case there are infinitely many variables (of $\mathcal{L}$ ) that have no free occurrence in its members, as well as infinitely many predicate letters of degree 1 that do not occur in its members. It has the $\forall$-property if and only if, for every wff $(\forall \mathbf{x}) \mathbf{A}$, it contains $(\forall \mathbf{x}) \neg[\mathbf{A} \rightarrow \mathbf{A}]$ or else there is a variable $\mathbf{y}$, not occurring in $\mathbf{A}$, and a predicate letter $\mathbf{F}$ of degree 1 such that $\Lambda$ contains both $\dot{\mathbf{S}}_{\mathbf{y}}^{\mathbf{x}} \mathbf{A} \rightarrow(\forall \mathbf{x}) \mathbf{A}$ and $\mathbf{F y}$. $\Lambda$ has the $\square \forall$-property if and only if it has the $\forall$-property and moreover, for every wff $\square\left[\mathbf{A}_{1} \rightarrow \cdots \square\left[\mathbf{A}_{m} \rightarrow \square(\forall \mathbf{x}) \mathbf{B}\right] \ldots\right]$ where $m \geq 0$, there is a variable $\mathbf{y}$, not occurring in any one of $\mathbf{A}_{1}, \ldots, \mathbf{A}_{m}, \mathbf{B}$, and a predicate letter $\mathbf{F}$ of degree 1 such that 
$\Lambda$ contains

$$
\begin{aligned}
\square\left[\mathbf{A}_{1} \rightarrow \cdots \square\left[\mathbf{A}_{m} \rightarrow \square\left[\mathbf{F y} \rightarrow \dot{\mathbf{S}}_{\mathbf{y}}^{\mathbf{x}} \mathbf{B}\right]\right]\right. & \ldots] \\
& \rightarrow \square\left[\mathbf{A}_{1} \rightarrow \ldots \square\left[\mathbf{A}_{m} \rightarrow \square(\forall \mathbf{x}) \mathbf{B}\right] \ldots\right] .
\end{aligned}
$$

Finally, $\Lambda$ has the $\square^{n}$-property just in case, for each $n$, there is a predicate letter $\mathbf{F}$ of degree 1 such that $\square^{n}\left(\forall x_{1}\right) \mathbf{F} x_{1} \in \Lambda$.

A set $W$ is $S$-full if and only if every member $\Gamma$ of $W$ satisfies the following conditions: it is a maximal $S$-consistent* set of wffs that has the $\forall$-property and, for some $\mathbf{F}$, contains $\left(\forall x_{1}\right) \mathbf{F} x_{1}$, and for each wff $\neg \square \mathbf{A} \in \Gamma$ there is a set $\Gamma^{\prime} \in W$ which contains $\neg \mathbf{A}$ and includes $\{\mathbf{B}: \square \mathbf{B} \in \Gamma\}$. ${ }^{7} \quad$ We can also define what it means to say that, in an $S$-full set $W$, a member $\Gamma^{\prime}$ is $n$ steps away from a member $\Gamma: \Gamma^{\prime}$ is 0 steps away if and only if it is $\Gamma$ itself, and it is $n+1$ steps away just in case a set $\Gamma^{\prime \prime} \in W$ is $n$ steps away from $\Gamma$ and $\left\{\mathbf{A}: \square \mathbf{A} \in \Gamma^{\prime \prime}\right\} \subseteq \Gamma^{\prime}$. Moreover, given an $S$-full set $W$ and a member $\Gamma$ of $W$, we can say that $\Gamma$ is distinguished in $W$ if and only if, for each $\Gamma^{\prime} \in W$, there is a number $n$ such that $\Gamma^{\prime}$ is $n$ steps away from $\Gamma$ and, for every $\mathbf{A}, @ \mathbf{A} \in \Gamma^{\prime}$ just in case $\mathbf{A} \in \Gamma$.

With the help of the above definitions we can turn to weak completeness. The main metatheorems here will be (3.11) and (3.14). (3.12) and (3.13) are in effect lemmas that contribute to demonstrating (3.14). (3.17), which is the central result about weak completeness for systems without identity, is deduced from (3.16) and (3.15), which is a corollary of (3.11) and (3.14). When we later discuss weak completeness for systems with identity, we shall see that (3.32), which is the central result for such systems, is deduced from (3.31) and (3.30), which is another corollary of (3.11) and (3.14). The proof of (3.12) - (3.14) adapts a method that is due to R. Thomason and is presented in Hughes and Cresswell [5], pp. 296-301. If instead we used the method of demonstrating completeness which was introduced in [7], the proof would be more complicated.

Metatheorem 3.11 If $\Lambda$ is an $S$-consistent, finite*, and nonempty actuality set of wffs, then there is an S-consistent* and finite* actuality set $\Lambda^{\prime}$ of wffs where $\Lambda \subseteq \Lambda^{\prime}$ and, for each individual constant $\mathbf{a}$, there is an atomic wff $\mathbf{A}$ such that $@ \mathbf{A} \in \Lambda^{\prime}$ and a occurs in $\mathbf{A}$.

Proof Let $\mathbf{F}_{1}, \mathbf{F}_{2}, \ldots$ be infinitely many distinct predicate letters of degree 1, excluding those that occur in members of $\Lambda$ and also excluding infinitely many others. $\Lambda^{\prime}$ will be $\Lambda \cup\left\{@ \mathbf{F}_{1} a_{1}, @ \mathbf{F}_{2} a_{2}, \ldots\right\}$. It is clear that $\Lambda^{\prime}$ has all the desirable features other than $S$-consistency*. So suppose, for reductio, that $\Lambda^{\prime}$ is not $S$-consistent*. Then

$$
\vdash_{S}^{*} \neg\left[@ \mathbf{G}_{1} \mathbf{a}_{1} \wedge \cdots \wedge @ \mathbf{G}_{n} \mathbf{a}_{n} \wedge \mathbf{A}_{1} \wedge \cdots \wedge \mathbf{A}_{i}\right]
$$

where $@ \mathbf{G}_{1} \mathbf{a}_{1}, \ldots, @ \mathbf{G}_{n} \mathbf{a}_{n}$ are distinct members of $\left\{@ \mathbf{F}_{1} a_{1}, @ \mathbf{F}_{2} a_{2}, \ldots\right\}$ while $\mathbf{A}_{1}, \ldots, \mathbf{A}_{i} \in \Lambda$. Hence,

$$
\vdash_{S} @ \mathbf{G}_{1} \mathbf{a}_{1} \rightarrow\left[\cdots \rightarrow\left[@ \mathbf{G}_{n} \mathbf{a}_{n} \rightarrow \neg\left[\mathbf{A}_{1} \wedge \cdots \wedge \mathbf{A}_{i}\right]\right] \ldots\right] .
$$

So this theorem has a proof in which @KA10-11 are not used. Let $\mathbf{y}_{1}, \ldots, \mathbf{y}_{n}$ be distinct variables that do not occur in the proof. If throughout that proof we replace $\mathbf{a}_{1}, \ldots, \mathbf{a}_{n}$ with $\mathbf{y}_{1}, \ldots, \mathbf{y}_{n}$, the result will again be a proof in which @ KA10-11 are not used. Thus

$$
\vdash_{S}^{*} @ \mathbf{G}_{1} \mathbf{y}_{1} \rightarrow\left[\cdots \rightarrow\left[@ \mathbf{G}_{n} \mathbf{y}_{n} \rightarrow \mathbf{S}_{\mathbf{y}_{1}, \ldots, \mathbf{y}_{n}}^{\mathbf{a}_{1}, \ldots, \mathbf{a}_{n}} \neg\left[\mathbf{A}_{1} \wedge \cdots \wedge \mathbf{A}_{i}\right]\right] \ldots\right] .
$$


Hence, by universal generalization, @ KA8, and ST9*,

$$
\begin{aligned}
\vdash_{S}^{*}\left(\forall \mathbf{y}_{1}\right) @ \mathbf{G}_{1} \mathbf{y}_{1} \rightarrow\left[@ \mathbf{G}_{2} \mathbf{y}_{2} \rightarrow[\cdots\right. \\
\left.\left.\quad \rightarrow\left[@ \mathbf{G}_{n} \mathbf{y}_{n} \rightarrow\left(\forall \mathbf{y}_{1}\right) \mathbf{S}_{\mathbf{y}_{1}, \ldots, \mathbf{y}_{n}}^{\mathbf{a}_{1}, \ldots, \mathbf{a}_{n}}\left[\mathbf{A}_{1} \wedge \cdots \wedge \mathbf{A}_{i}\right]\right] \ldots\right]\right] .
\end{aligned}
$$

Therefore, by @KR3, @KA3, and @KA5,

$$
\begin{aligned}
\vdash_{S} @\left(\forall \mathbf{y}_{1}\right) @ \mathbf{G}_{1} \mathbf{y}_{1} & \rightarrow\left[@ \mathbf{G}_{2} \mathbf{y}_{2} \rightarrow[\cdots\right. \\
& \left.\left.\rightarrow\left[@ \mathbf{G}_{n} \mathbf{y}_{n} \rightarrow @\left(\forall \mathbf{y}_{1}\right) \mathbf{S}_{\mathbf{y}_{1}, \ldots, \mathbf{y}_{n}}^{\mathbf{a}_{1}, \ldots, \mathbf{a}_{n}} \neg\left[\mathbf{A}_{1} \wedge \cdots \wedge \mathbf{A}_{i}\right]\right] \ldots\right]\right] .
\end{aligned}
$$

So, by $S \mathrm{~T} 17^{*}$ and @KR5,

$$
\vdash_{S}^{*} @ \mathbf{G}_{2} \mathbf{y}_{2} \rightarrow\left[\cdots \rightarrow\left[@ \mathbf{G}_{n} \mathbf{y}_{n} \rightarrow @\left(\forall \mathbf{y}_{1}\right) \mathbf{S}_{\mathbf{y}_{1}, \ldots, \mathbf{y}_{n}}^{\mathbf{a}_{1}, \ldots, \mathbf{a}_{n}} \neg\left[\mathbf{A}_{1} \wedge \cdots \wedge \mathbf{A}_{i}\right]\right] \ldots\right] .
$$

Proceeding in the same way, we infer that

$$
\vdash_{S}^{*} @\left(\forall \mathbf{y}_{n}\right) \cdots @\left(\forall \mathbf{y}_{1}\right) \mathbf{S}_{\mathbf{y}_{1}, \ldots, \mathbf{y}_{n}}^{\mathbf{a}_{1}, \ldots, \mathbf{a}_{n}} \neg\left[\mathbf{A}_{1} \wedge \cdots \wedge \mathbf{A}_{i}\right]
$$

Hence, by $\left.@ K A 10, \vdash_{S}\left(\forall \mathbf{y}_{n}\right) @\left(\forall \mathbf{y}_{n-1}\right) \cdots @\left(\forall \mathbf{y}_{1}\right) \mathbf{S}_{\mathbf{y}_{1}, \ldots, \mathbf{y}_{n}}^{\mathbf{a}_{1}, \ldots}, \mathbf{a}_{n} \mathbf{A}_{1} \wedge \cdots \wedge \mathbf{A}_{i}\right]$ and so, by $\left.@ K A 11, \vdash_{S} @\left(\forall \mathbf{y}_{n-1}\right) \cdots @\left(\forall \mathbf{y}_{1}\right) \mathbf{S}_{\mathbf{y}_{1}, \ldots, \mathbf{y}_{n-1}}^{\mathbf{a}_{1}, \ldots} \mathbf{a}_{1} \wedge \cdots \wedge \mathbf{A}_{i}\right]$. Continuing in the same manner, we conclude that $\vdash_{S} \neg\left[\mathbf{A}_{1} \wedge \cdots \wedge \mathbf{A}_{i}\right]$, which contradicts the $S$-consistency of $\Lambda$.

Metatheorem 3.12 If $\Lambda$ is an $S$-consistent ${ }^{*}$, finite ${ }^{*}$, and nonempty actuality set of wffs, then there is a maximal $S$-consistent* set $\Gamma$ of $w f f s$ which is a superset of $\Lambda$, has the $\square \forall$-property and the $\square^{n}$-property, and includes $\{@ \mathbf{A}: \mathbf{A} \in \Gamma\}$.

Proof Let $\mathbf{F}_{0}, \mathbf{F}_{1}, \ldots$ be infinitely many distinct predicate letters of degree 1, excluding those that occur in members of $\Lambda$ and also excluding infinitely many others. $\Lambda^{\prime}$ will be $\Lambda \cup\left\{@\left(\forall x_{1}\right) \mathbf{F}_{0} x_{1}, @ \square\left(\forall x_{1}\right) \mathbf{F}_{1} x_{1}\right.$, @ $\left.\square\left(\forall x_{1}\right) \mathbf{F}_{2} x_{1}, \ldots\right\}$. The $S$ consistency* of $\Lambda^{\prime}$ can be proved using @KR5. $\Lambda^{\prime}$ is also a finite* actuality set.

Now let $\mathbf{C}_{1}, \mathbf{C}_{2}, \ldots$ be an enumeration of all the wffs that have the form @ $\mathbf{A}$ or the form $(\forall \mathbf{x}) \mathbf{A}$ or the form $\square\left[\mathbf{A}_{1} \rightarrow \cdots \square\left[\mathbf{A}_{m} \rightarrow \square(\forall \mathbf{x}) \mathbf{B}\right] \ldots\right]$. We shall define the sets $\Gamma_{0}, \Gamma_{1}, \Gamma_{2}, \ldots$ where $\Gamma_{0}$ is $\Lambda^{\prime}$.

Case $1 \mathbf{C}_{n+1}$ is @ A.

Subcase 1a There are wffs $\mathbf{B}_{1}, \ldots, \mathbf{B}_{i} \in \Gamma_{n}$ such that ${ }^{*}{ }_{S} \mathbf{B}_{1} \wedge \cdots \wedge \mathbf{B}_{i} \rightarrow @ \mathbf{A}$. Then $\Gamma_{n+1}$ will be $\Gamma_{n} \cup\{@ \mathbf{A}\}$.

Subcase 1b There are no such wffs $\mathbf{B}_{1}, \ldots, \mathbf{B}_{i}$. Then $\Gamma_{n+1}$ will be $\Gamma_{n} \cup\{@ \neg \mathbf{A}\}$.

Case $2 \mathbf{C}_{n+1}$ is $(\forall \mathbf{x}) \mathbf{A}$.

Subcase 2a There are wffs $\mathbf{B}_{1}, \ldots, \mathbf{B}_{i} \in \Gamma_{n}$ such that $\vdash_{S}^{*} \mathbf{B}_{1} \wedge \cdots \wedge \mathbf{B}_{i}$ $\rightarrow @(\forall \mathbf{x}) \neg[\mathbf{A} \rightarrow \mathbf{A}]$. Then $\Gamma_{n+1}$ will be $\Gamma_{n} \cup\{@(\forall \mathbf{x}) \neg[\mathbf{A} \rightarrow \mathbf{A}]\}$.

Subcase 2b There are no such wffs $\mathbf{B}_{1}, \ldots, \mathbf{B}_{i}$. Then $\Gamma_{n+1}$ will be $\Gamma_{n} \cup\{@ \mathbf{F y}$, $\left.@\left[\dot{\mathbf{S}}_{\mathbf{y}}^{\mathbf{x}} \mathbf{A} \rightarrow(\forall \mathbf{x}) \mathbf{A}\right]\right\}$, where $\mathbf{y}$ is the first variable (in alphabetical order) that has no free occurrence in any member of $\Gamma_{n}$ and does not occur in $\mathbf{A}$, and $\mathbf{F}$ is the first predicate letter of degree 1 (in alphabetical order) that occurs neither in the members of $\Gamma_{n}$ nor in $\mathbf{A}$.

Case $3 \mathbf{C}_{n+1}$ is $\square\left[\mathbf{A}_{1} \rightarrow \cdots \square\left[\mathbf{A}_{m} \rightarrow \square(\forall \mathbf{x}) \mathbf{B}\right] \ldots\right]$. Then $\Gamma_{n+1}$ will be $\Gamma_{n} \cup\left\{@ \mathbf{C}^{\prime}\right\}, \mathbf{C}^{\prime}$ being the wff $\square\left[\mathbf{A}_{1} \rightarrow \cdots \square\left[\mathbf{A}_{m} \rightarrow \square\left[\mathbf{F y} \rightarrow \dot{\mathbf{S}}_{\mathbf{y}}^{\mathbf{x}} \mathbf{B}\right]\right] \ldots\right] \rightarrow \mathbf{C}_{n+1}$, where $\mathbf{y}$ is the first variable that has no free occurrence in any member of $\Gamma_{n}$ and 
does not occur in $\mathbf{A}_{1}, \ldots, \mathbf{A}_{m}, \mathbf{B}$, while $\mathbf{F}$ is the first predicate letter of degree 1 that occurs neither in the members of $\Gamma_{n}$ nor in $\mathbf{A}_{1}, \ldots, \mathbf{A}_{m}, \mathbf{B}$.

Assume for reductio that $\Gamma_{n}$ is $S$-consistent* but $\Gamma_{n+1}$ is not. Subcases 1a and 2a are trivial, and Subcase $1 \mathrm{~b}$ follows from $S \mathrm{~T} 4^{*}$.

Subcase $2 \mathrm{~b}$. There will be wffs $\mathbf{D}_{1}, \ldots, \mathbf{D}_{j} \in \Gamma_{n}$ such that

$$
\vdash_{S}^{*} \mathbf{D}_{1} \wedge \cdots \wedge \mathbf{D}_{j} \rightarrow\left[\left[\mathbf{F y} \rightarrow \neg @\left[\dot{\mathbf{S}}_{\mathbf{y}}^{\mathbf{x}} \mathbf{A} \rightarrow(\forall \mathbf{x}) \mathbf{A}\right]\right] .\right.
$$

Hence, by @KA4 and $S T 2 *$ (inter alia),

$$
\vdash_{S}^{*} \mathbf{D}_{1} \wedge \cdots \wedge \mathbf{D}_{j} \rightarrow @\left[\mathbf{F y} \rightarrow \dot{\mathbf{S}}_{\mathbf{y}}^{\mathbf{x}} \mathbf{A} \wedge \neg(\forall \mathbf{x}) \mathbf{A}\right] .
$$

Thus, by@KR4, @KA8, and ST13*,

$$
\vdash_{S}^{*} \mathbf{D}_{1} \wedge \cdots \wedge \mathbf{D}_{j} \rightarrow @\left[(\forall \mathbf{y}) \mathbf{F y} \rightarrow(\forall \mathbf{y}) \dot{\mathbf{S}}_{\mathbf{y}}^{\mathbf{x}} \mathbf{A} \wedge(\forall \mathbf{y}) \neg(\forall \mathbf{x}) \mathbf{A}\right] .
$$

So, by @KA3, ST11*, and $S \mathrm{~T} 14^{*}$,

$$
\vdash_{S} @(\forall \mathbf{y}) \mathbf{F y} \rightarrow\left[\mathbf{D}_{1} \wedge \cdots \wedge \mathbf{D}_{j} \rightarrow @[(\forall \mathbf{x}) \mathbf{A} \wedge[\neg(\forall \mathbf{x}) \mathbf{A} \vee(\forall \mathbf{x}) \neg[\mathbf{A} \rightarrow \mathbf{A}]]]\right] .
$$

Therefore, by @KR5, $\vdash_{S}^{*} \mathbf{D}_{1} \wedge \cdots \wedge \mathbf{D}_{j} \rightarrow @(\forall \mathbf{x}) \neg[\mathbf{A} \rightarrow \mathbf{A}]$, which contradicts the characteristics of the subcase.

Case 3. There will be wffs $\mathbf{D}_{1}, \ldots, \mathbf{D}_{j} \in \Gamma_{n}$ such that $\vdash_{S}{ }_{S} \mathbf{D}_{1} \wedge \cdots \wedge \mathbf{D}_{j}$ $\rightarrow \neg @ \mathbf{C}^{\prime}$. Hence, by @KA4 and $S \mathrm{~T} 7^{*}$,

$$
\vdash_{S}^{*} \mathbf{D}_{1} \wedge \cdots \wedge \mathbf{D}_{j} \rightarrow @ \square\left[\mathbf{A}_{1} \rightarrow \cdots \square\left[\mathbf{A}_{m} \rightarrow \square\left[\mathbf{F y} \rightarrow \dot{\mathbf{S}}_{\mathbf{y}}^{\mathbf{x}} \mathbf{B}\right]\right] \ldots\right]
$$

and

$$
\vdash_{S}^{*} \mathbf{D}_{1} \wedge \cdots \wedge \mathbf{D}_{j} \rightarrow \neg @ \square\left[\mathbf{A}_{1} \rightarrow \cdots \square\left[\mathbf{A}_{m} \rightarrow \square(\forall \mathbf{x}) \mathbf{B}\right] \ldots\right] .
$$

Thus, by $S R 8$ (or $S R 7$ ) and @KA8,

$$
\vdash_{S}^{*} \mathbf{D}_{1} \wedge \cdots \wedge \mathbf{D}_{j} \rightarrow @ \square\left[\mathbf{A}_{1} \rightarrow \cdots \square\left[\mathbf{A}_{m} \rightarrow \square\left[(\forall \mathbf{y}) \mathbf{F y} \rightarrow(\forall \mathbf{y}) \dot{\mathbf{S}}_{\mathbf{y}}^{\mathbf{x}} \mathbf{B}\right]\right] \ldots\right] .
$$

So, by @KA2,

$$
\begin{aligned}
\vdash_{S}^{*} & \mathbf{D}_{1} \wedge \cdots \wedge \mathbf{D}_{j} \\
\quad \rightarrow & @\left[\mathbf{A}_{1} \rightarrow \cdots \square\left[\mathbf{A}_{m-1} \rightarrow \square\left[\square(\forall \mathbf{y}) \mathbf{F y} \rightarrow\left[\mathbf{A}_{m} \rightarrow \square(\forall \mathbf{y}) \dot{\mathbf{S}}_{\mathbf{y}}^{\mathbf{x}} \mathbf{B}\right]\right]\right] \ldots\right] .
\end{aligned}
$$

Proceeding repeatedly as in the last step, we infer that ${\vdash_{S}}_{S} @ \square^{m+1}(\forall \mathbf{y}) \mathbf{F y} \rightarrow\left[\mathbf{D}_{1} \wedge \cdots \wedge \mathbf{D}_{j} \rightarrow @ \square\left[\mathbf{A}_{1} \rightarrow \cdots \square\left[\mathbf{A}_{m} \rightarrow \square(\forall \mathbf{y}) \dot{\mathbf{S}}_{\mathbf{y}}^{\mathbf{x}} \mathbf{B}\right] \ldots\right]\right]$.

Therefore, by @KR5 and ST11*,

$$
\vdash_{S}^{*} \mathbf{D}_{1} \wedge \cdots \wedge \mathbf{D}_{j} \rightarrow @ \square\left[\mathbf{A}_{1} \rightarrow \cdots \square\left[\mathbf{A}_{m} \rightarrow \square(\forall \mathbf{x}) \mathbf{B}\right] \ldots\right] .
$$

Hence $\vdash_{S}^{*} \neg\left[\mathbf{D}_{1} \wedge \cdots \wedge \mathbf{D}_{j}\right]$, which contradicts the $S$-consistency* of $\Gamma_{n}$.

Thus $\Gamma_{0}, \Gamma_{1}, \Gamma_{2}, \ldots$ are all $S$-consistent*, as is their union, $\Gamma^{\prime} . \Gamma^{\prime}$ is also an actuality set.

$\Gamma$ will be $\Gamma^{\prime} \cup\left\{\mathbf{A}: @ \mathbf{A} \in \Gamma^{\prime}\right\}$. Assume that $\Gamma$ is not $S$-consistent*. Then $*_{S}^{*} \neg\left[\mathbf{B}_{1} \wedge \cdots \wedge \mathbf{B}_{i} \wedge \mathbf{A}_{1} \wedge \cdots \wedge \mathbf{A}_{j}\right]$ where $\mathbf{B}_{1}, \ldots, \mathbf{B}_{i}, @ \mathbf{A}_{1}, \ldots, @ \mathbf{A}_{j} \in \Gamma^{\prime}$. Hence, by @KR3 and @KA4, $\vdash_{S} \neg @\left[\mathbf{B}_{1} \wedge \cdots \wedge \mathbf{B}_{i} \wedge \mathbf{A}_{1} \wedge \cdots \wedge \mathbf{A}_{j}\right]$. So, by $S T 7^{*}, \vdash_{S}^{*} \neg\left[@ \mathbf{B}_{1} \wedge \cdots \wedge @ \mathbf{B}_{i} \wedge \mathbf{B}_{1} \wedge \cdots \wedge @ \mathbf{A}_{j}\right]$. Thus, by @KA5, $\vdash_{S}^{*} \neg\left[\mathbf{B}_{1} \wedge \cdots \wedge \mathbf{B}_{i} \wedge @ \mathbf{A}_{1} \wedge \cdots \wedge @ \mathbf{A}_{j}\right]$, which contradicts the $S$-consistency* of $\Gamma^{\prime} . \Gamma$ is therefore $S$-consistent*. It is also maximal and has the $\square \forall$-property and the $\square^{n}$-property. Finally, if $\mathbf{A} \in \Gamma$ then $@ \mathbf{A} \in \Gamma$; for if $@ \mathbf{A} \notin \Gamma$, then $@ \neg \mathbf{A} \in \Gamma^{\prime}$ and so $\neg \mathbf{A} \in \Gamma$. 
In order to prove the next metatheorem, we must presuppose an enumeration of all the ordered pairs $\langle\mathbf{F}, \mathbf{y}\rangle$ where $\mathbf{F}$ is a predicate letter of degree 1 and $\mathbf{y}$ is a variable.

Metatheorem 3.13 Let $\Gamma$ and $\Delta$ be sets of wffs such that $\Delta$ is maximal and $S$ consistent $^{*}$, has the $\square \forall$-property and the $\square^{n}$-property, and includes $\{@ \mathbf{B}: \mathbf{B} \in \Gamma\}$. Also let $\square \mathbf{A} \notin \Delta$. Then there is a maximal $S$-consistent* set $\Theta$ of wffs which has the $\square \forall$-property and the $\square^{n}$-property, includes $\{@ \mathbf{B}: \mathbf{B} \in \Gamma\}$, and is such that $\neg \mathbf{A} \in \Theta$ and $\{\mathbf{C}: \square \mathbf{C} \in \Delta\} \subseteq \Theta$.

Proof Let $\mathbf{D}_{1}, \mathbf{D}_{2}, \ldots$ be an enumeration of all the wffs that have the form $(\forall \mathbf{x}) \mathbf{B}$ or the form $\square\left[\mathbf{A}_{1} \rightarrow \cdots \square\left[\mathbf{A}_{m} \rightarrow \square(\forall \mathbf{x}) \mathbf{B}\right] \ldots\right]$. We shall define the sets $\Theta_{0}, \Theta_{1}, \Theta_{2}, \ldots$

$\Theta_{0}$ will be $\{\neg \mathbf{A}\} \cup\{@ \mathbf{B}: \mathbf{B} \in \Gamma\} \cup\{\mathbf{C}: \square \mathbf{C} \in \Delta\}$. Assume that $\Theta_{0}$ is not $S$-consistent*. Then, for some wffs $\square \mathbf{C}_{1}, \ldots, \square \mathbf{C}_{i} \in \Delta$ and $\mathbf{B}_{1}, \ldots, \mathbf{B}_{l} \in \Gamma$, ${ }^{*}{ }_{S} \mathbf{C}_{1} \wedge \cdots \wedge \mathbf{C}_{i} \wedge @ \mathbf{B}_{1} \wedge \cdots \wedge @ \mathbf{B}_{l} \rightarrow \mathbf{A}$. Hence, by $@ \mathrm{KR} 2$, @KA2, and $S \mathrm{~T} 1^{*}, \vdash^{*}{ }_{S} \square \mathbf{C}_{1} \wedge \cdots \wedge \square \mathbf{C}_{i} \wedge \square @ \mathbf{B}_{1} \wedge \cdots \wedge \square @ \mathbf{B}_{l} \rightarrow \square \mathbf{A}$. Thus, by @KA6, $\vdash_{S} \square \mathbf{C}_{1} \wedge \cdots \wedge \square \mathbf{C}_{i} \wedge @ \mathbf{B}_{1} \wedge \cdots \wedge @ \mathbf{B}_{l} \rightarrow \square \mathbf{A}$. So, by (3.10), $\square \mathbf{A} \in \Delta$, which is contrary to our hypothesis. $\Theta_{0}$ is therefore $S$-consistent*. Note that $\Theta_{0}$ also has the $\square^{n}$-property.

Case $1 \quad \mathbf{D}_{n+1}$ is $(\forall \mathbf{x}) \mathbf{B}$.

Subcase 1a There are wffs $\mathbf{E}_{1}, \ldots, \mathbf{E}_{i} \in \Theta_{n}$ such that $\vdash^{*} \mathbf{E}_{1} \wedge \cdots \wedge \mathbf{E}_{i}$ $\rightarrow(\forall \mathbf{x}) \neg[\mathbf{B} \rightarrow \mathbf{B}]$. Then $\Theta_{n+1}$ will be $\Theta_{n} \cup\{(\forall \mathbf{x}) \neg[\mathbf{B} \rightarrow \mathbf{B}]\}$.

Subcase 1b There are no such wffs $\mathbf{E}_{1}, \ldots, \mathbf{E}_{i}$. Then $\Theta_{n+1}$ will be $\Theta_{n} \cup\{\mathbf{F y}$, $\left.\dot{\mathbf{S}}_{\mathbf{y}}^{\mathbf{x}} \mathbf{B} \rightarrow(\forall \mathbf{x}) \mathbf{B}\right\}$ where $\langle\mathbf{F}, \mathbf{y}\rangle$ is the first ordered pair of a predicate letter $\mathbf{G}$ of degree 1 and a variable $\mathbf{z}$ such that $\mathbf{z}$ does not occur in $\mathbf{B}$ and $\Theta_{n} \cup\left\{\mathbf{G z}, \dot{\mathbf{S}}_{\mathbf{z}}^{\mathbf{x}} \mathbf{B} \rightarrow(\forall \mathbf{x}) \mathbf{B}\right\}$ is $S$-consistent*.

Case $2 \mathbf{D}_{n+1}$ is $\square\left[\mathbf{A}_{1} \rightarrow \cdots \square\left[\mathbf{A}_{m} \rightarrow \square(\forall \mathbf{x}) \mathbf{B}\right] \ldots\right]$. Then $\Theta_{n+1}$ will be $\Theta_{n} \cup\left\{\square\left[\mathbf{A}_{1} \rightarrow \cdots \square\left[\mathbf{A}_{m} \rightarrow \square\left[\mathbf{F y} \rightarrow \dot{\mathbf{S}}_{\mathbf{y}}^{\mathbf{x}} \mathbf{B}\right]\right] \ldots\right] \rightarrow \mathbf{D}_{n+1}\right\}$ where $\langle\mathbf{F}, \mathbf{y}\rangle$ is the first ordered pair of a predicate letter $\mathbf{G}$ of degree 1 and a variable $\mathbf{z}$ such that $\mathbf{z}$ does not occur in $\mathbf{A}_{1}, \ldots, \mathbf{A}_{m}, \mathbf{B}$ and $\Theta_{n} \cup\left\{\square\left[\mathbf{A}_{1} \rightarrow \cdots \square\left[\mathbf{A}_{m} \rightarrow \square\left[\mathbf{G z} \rightarrow \dot{\mathbf{S}}_{\mathbf{z}}^{\mathbf{x}} \mathbf{B}\right]\right] \ldots\right]\right.$ $\left.\rightarrow \mathbf{D}_{n+1}\right\}$ is $S$-consistent*

Assume that $\Theta_{n}$ is defined and $S$-consistent*. If we have Subcase 1a, it is then trivial that $\Theta_{n+1}$ is defined and $S$-consistent*.

Subcase 1b. Suppose that, for every predicate letter $\mathbf{G}$ of degree 1 and every variable $\mathbf{z}$ not occurring in $\mathbf{B}, \Theta_{n} \cup\left\{\mathbf{G z}, \dot{\mathbf{S}}_{\mathbf{z}}^{\mathbf{x}} \mathbf{B} \rightarrow(\forall \mathbf{x}) \mathbf{B}\right\}$ is not $S$-consistent*. If $\mathbf{G}$ and $\mathbf{z}$ have this feature, then, for some wffs $\square \mathbf{C}_{1}, \ldots, \square \mathbf{C}_{i} \in \Delta$ and $\mathbf{B}_{1}, \ldots, \mathbf{B}_{l} \in \Gamma$,

$$
\vdash_{S}^{*} \mathbf{C}_{1} \wedge \cdots \wedge \mathbf{C}_{i} \wedge @ \mathbf{B}_{1} \wedge \cdots \wedge @ \mathbf{B}_{l} \rightarrow\left[\mathbf{E} \rightarrow\left[\mathbf{G z} \rightarrow \dot{\mathbf{S}}_{\mathbf{z}}^{\mathbf{x}} \mathbf{B} \wedge \neg(\forall \mathbf{x}) \mathbf{B}\right]\right]
$$

where $\mathbf{E}$ is the conjunction (in some order) of $\neg \mathbf{A}$ and all wffs that our construction has added to $\Theta_{0}$ at stages $1, \ldots, n$. Hence

$\vdash_{S}^{*} \square \mathbf{C}_{1} \wedge \cdots \wedge \square \mathbf{C}_{i} \wedge \square @ \mathbf{B}_{1} \wedge \cdots \wedge \square @ \mathbf{B}_{l} \rightarrow \square\left[\mathbf{E} \rightarrow\left[\mathbf{G z} \rightarrow \dot{\mathbf{S}}_{\mathbf{z}}^{\mathbf{x}} \mathbf{B} \wedge \neg(\forall \mathbf{x}) \mathbf{B}\right]\right]$.

So

$$
\vdash_{S}^{*} \square \mathbf{C}_{1} \wedge \cdots \wedge \square \mathbf{C}_{i} \wedge @ \mathbf{B}_{1} \wedge \cdots \wedge @ \mathbf{B}_{l} \rightarrow \square\left[\mathbf{G z} \rightarrow\left[\mathbf{E} \rightarrow \dot{\mathbf{S}}_{\mathbf{z}}^{\mathbf{x}} \mathbf{B} \wedge \neg(\forall \mathbf{x}) \mathbf{B}\right]\right] .
$$

Thus by (3.10), for every $\mathbf{G}$ of degree 1 and every $\mathbf{z}$ not occurring in $\mathbf{B}$, the wff $\square\left[\mathbf{G z} \rightarrow\left[\mathbf{E} \rightarrow \dot{\mathbf{S}}_{\mathbf{z}}^{\mathbf{x}} \mathbf{B} \wedge \neg(\forall \mathbf{x}) \mathbf{B}\right]\right]$ belongs to $\Delta$. 
Let $\mathbf{v}$ be a variable that occurs neither in $\mathbf{E}$ nor in $\mathbf{B}$. Since $\Delta$ has the $\square \forall$ property, there is a predicate letter $\mathbf{G}$ of degree 1 and a variable $\mathbf{z}$ not occurring in $\mathbf{E} \rightarrow \dot{\mathbf{S}}_{\mathbf{v}}^{\mathbf{x}} \mathbf{B} \wedge \neg(\forall \mathbf{x}) \mathbf{B}$ such that $\Delta$ contains

$$
\square\left[\mathbf{G z} \rightarrow\left[\mathbf{E} \rightarrow \dot{\mathbf{S}}_{\mathbf{z}}^{\mathbf{x}} \mathbf{B} \wedge \neg(\forall \mathbf{x}) \mathbf{B}\right]\right] \rightarrow \square(\forall \mathbf{v})\left[\mathbf{E} \rightarrow \dot{\mathbf{S}}_{\mathbf{v}}^{\mathbf{x}} \mathbf{B} \wedge \neg(\forall \mathbf{x}) \mathbf{B}\right] .
$$

Hence $\Delta$ contains $\square(\forall \mathbf{v})\left[\mathbf{E} \rightarrow \dot{\mathbf{S}}_{\mathbf{v}}^{\mathbf{x}} \mathbf{B} \wedge \neg(\forall \mathbf{x}) \mathbf{B}\right]$. But

$$
\begin{aligned}
& \vdash_{S}^{*} \square(\forall \mathbf{v})\left[\mathbf{E} \rightarrow \dot{\mathbf{S}}_{\mathbf{v}}^{\mathbf{x}} \mathbf{B} \wedge\right.\neg(\forall \mathbf{x}) \mathbf{B}] \rightarrow \square\left[\mathbf{E} \rightarrow(\forall \mathbf{v}) \dot{\mathbf{S}}_{\mathbf{v}}^{\mathbf{x}} \mathbf{B} \wedge(\forall \mathbf{v}) \neg(\forall \mathbf{x}) \mathbf{B}\right] \\
& \vdash^{*}{ }_{S} \square\left[\mathbf{E} \rightarrow(\forall \mathbf{v}) \dot{\mathbf{S}}_{\mathbf{v}}^{\mathbf{x}} \mathbf{B} \wedge(\forall \mathbf{v}) \neg(\forall \mathbf{x}) \mathbf{B}\right] \\
& \rightarrow \square[\mathbf{E} \rightarrow(\forall \mathbf{x}) \mathbf{B} \wedge[\neg(\forall \mathbf{x}) \mathbf{B} \vee(\forall \mathbf{x}) \neg[\mathbf{B} \rightarrow \mathbf{B}]]]
\end{aligned}
$$

and

$$
\vdash_{S}^{*} \square[\mathbf{E} \rightarrow(\forall \mathbf{x}) \mathbf{B} \wedge[\neg(\forall \mathbf{x}) \mathbf{B} \vee(\forall \mathbf{x}) \neg[\mathbf{B} \rightarrow \mathbf{B}]]] \rightarrow \square[\mathbf{E} \rightarrow(\forall \mathbf{x}) \neg[\mathbf{B} \rightarrow \mathbf{B}]] .
$$

Thus $\Delta$ contains $\square[\mathbf{E} \rightarrow(\forall \mathbf{x}) \neg[\mathbf{B} \rightarrow \mathbf{B}]]$. So $\Theta_{0}$, as well as $\Theta_{n}$, contains $\mathbf{E} \rightarrow(\forall \mathbf{x}) \neg[\mathbf{B} \rightarrow \mathbf{B}]$, contrary to the characteristics of the subcase.

Hence, our supposition that, for every predicate letter $\mathbf{G}$ of degree 1 and every variable $\mathbf{z}$ not occurring in $\mathbf{B}, \Theta_{n} \cup\left\{\mathbf{G z}, \dot{\mathbf{S}}_{\mathbf{z}}^{\mathbf{x}} \mathbf{B} \rightarrow(\forall \mathbf{x}) \mathbf{B}\right\}$ is not $S$-consistent* was wrong. $\Theta_{n+1}$ is therefore defined and $S$-consistent*.

Case 2. Suppose that, for every predicate letter $\mathbf{G}$ of degree 1 and every variable $\mathbf{z}$ not occurring in $\mathbf{A}_{1}, \ldots, \mathbf{A}_{m}, \mathbf{B}$, the set $\Theta_{n} \cup\left\{\square\left[\mathbf{A}_{1} \rightarrow \ldots \square\left[\mathbf{A}_{m}\right.\right.\right.$ $\left.\left.\left.\rightarrow \square\left[\mathbf{G z} \rightarrow \dot{\mathbf{S}}_{\mathbf{z}}^{\mathbf{x}} \mathbf{B}\right]\right] \ldots\right] \rightarrow \mathbf{D}_{n+1}\right\}$ is not $S$-consistent*. If $\mathbf{G}$ and $\mathbf{z}$ have this feature, then, for some wffs $\square \mathbf{C}_{1}, \ldots, \square \mathbf{C}_{i} \in \Delta$ and $\mathbf{B}_{1}, \ldots, \mathbf{B}_{l} \in \Gamma$,

$$
\begin{aligned}
\vdash_{S}^{*} \mathbf{C}_{1} \wedge \cdots \wedge \mathbf{C}_{i} \wedge @ \mathbf{B}_{1} & \wedge \cdots \wedge @ \mathbf{B}_{l} \\
& \rightarrow\left[\mathbf{E} \rightarrow \square\left[\mathbf{A}_{1} \rightarrow \cdots \square\left[\mathbf{A}_{m} \rightarrow \square\left[\mathbf{G z} \rightarrow \dot{\mathbf{S}}_{\mathbf{z}}^{\mathbf{x}} \mathbf{B}\right]\right] \ldots\right]\right]
\end{aligned}
$$

and

$$
\begin{aligned}
\vdash_{S}^{*} \mathbf{C}_{1} \wedge \cdots \wedge \mathbf{C}_{i} \wedge @ \mathbf{B}_{1} & \wedge \\
& \cdots \wedge @ \mathbf{B}_{l} \\
& \rightarrow\left[\mathbf{E} \rightarrow \neg \square\left[\mathbf{A}_{1} \rightarrow \cdots \square\left[\mathbf{A}_{m} \rightarrow \square(\forall \mathbf{x}) \mathbf{B}\right] \ldots\right]\right]
\end{aligned}
$$

where $\mathbf{E}$ is as in Subcase $1 \mathrm{~b}$ above. Hence

$$
\begin{aligned}
\vdash_{S}^{*} \square \mathbf{C}_{1} \wedge \cdots \wedge \square \mathbf{C}_{i} & \wedge @ \mathbf{B}_{1} \wedge \cdots \wedge @ \mathbf{B}_{l} \\
& \rightarrow \square\left[\mathbf{E} \rightarrow \square\left[\mathbf{A}_{1} \rightarrow \cdots \square\left[\mathbf{A}_{m} \rightarrow \square\left[\mathbf{G z} \rightarrow \dot{\mathbf{S}}_{\mathbf{z}}^{\mathbf{x}} \mathbf{B}\right]\right] \ldots\right]\right]
\end{aligned}
$$

and

$$
\begin{aligned}
\vdash_{S}^{*} \square \mathbf{C}_{1} \wedge \cdots \wedge \square \mathbf{C}_{i} \wedge @ \mathbf{B}_{1} \wedge \cdots \wedge @ \mathbf{B}_{l} \\
\rightarrow \square\left[\mathbf{E} \rightarrow \neg \square\left[\mathbf{A}_{1} \rightarrow \cdots \square\left[\mathbf{A}_{m} \rightarrow \square(\forall \mathbf{x}) \mathbf{B}\right] \ldots\right]\right] .
\end{aligned}
$$

Thus, for every $\mathbf{G}$ of degree 1 and every $\mathbf{z}$ not occurring in $\mathbf{A}_{1}, \ldots, \mathbf{A}_{m}, \mathbf{B}$, the wffs $\square\left[\mathbf{E} \rightarrow \square\left[\mathbf{A}_{1} \rightarrow \cdots \square\left[\mathbf{A}_{m} \rightarrow \square\left[\mathbf{G z} \rightarrow \dot{\mathbf{S}}_{\mathbf{z}}^{\mathbf{x}} \mathbf{B}\right]\right] \ldots\right]\right]$ and $\square\left[\mathbf{E} \rightarrow \neg \square\left[\mathbf{A}_{1}\right.\right.$ $\left.\left.\rightarrow \cdots \square\left[\mathbf{A}_{m} \rightarrow \square(\forall \mathbf{x}) \mathbf{B}\right] \ldots\right]\right]$ belong to $\Delta$.

Now, since $\Delta$ has the $\square \forall$-property, there is a predicate letter $\mathbf{G}$ of degree 1 and a variable $\mathbf{z}$ not occurring in $\mathbf{E}, \mathbf{A}_{1}, \ldots, \mathbf{A}_{m}, \mathbf{B}$ such that $\Delta$ contains

$$
\begin{aligned}
\square\left[\mathbf { E } \rightarrow \square \left[\mathbf { A } _ { 1 } \rightarrow \cdots \square \left[\mathbf{A}_{m}\right.\right.\right. & \left.\left.\left.\rightarrow \square\left[\mathbf{G z} \rightarrow \dot{\mathbf{S}}_{\mathbf{z}}^{\mathbf{x}} \mathbf{B}\right]\right] \ldots\right]\right] \\
& \rightarrow \square\left[\mathbf{E} \rightarrow \square\left[\mathbf{A}_{1} \rightarrow \cdots \square\left[\mathbf{A}_{m} \rightarrow \square(\forall \mathbf{x}) \mathbf{B}\right] \ldots\right]\right] .
\end{aligned}
$$


Hence $\Delta$ contains $\square\left[\mathbf{E} \rightarrow \square\left[\mathbf{A}_{1} \rightarrow \cdots \square\left[\mathbf{A}_{m} \rightarrow \square(\forall \mathbf{x}) \mathbf{B}\right] \ldots\right]\right]$. Since it also contains $\square\left[\mathbf{E} \rightarrow \neg \square\left[\mathbf{A}_{1} \rightarrow \cdots \square\left[\mathbf{A}_{m} \rightarrow \square(\forall \mathbf{x}) \mathbf{B}\right] \ldots\right]\right]$, we have that $\square \neg \mathbf{E} \in \Delta$. Thus, $\neg \mathbf{E}$ belongs to $\Theta_{0}$ and to $\Theta_{n}$, contrary to the $S$-consistency* of $\Theta_{n}$. Consequently, once more, $\Theta_{n+1}$ is defined and $S$-consistent*.

So, all of $\Theta_{0}, \Theta_{1}, \Theta_{2}, \ldots$ are defined and $S$-consistent*. Let $\Theta^{\prime}$ be their union. It is $S$-consistent*, too, and has the $\square \forall$-property. $\Theta^{\prime}$ can be extended in a standard manner to a maximal $S$-consistent* set $\Theta$. $\Theta$ has all the desirable features.

Metatheorem 3.14 If $\Lambda$ is an $S$-consistent ${ }^{*}$, finite ${ }^{*}$, and nonempty actuality set of wffs, then there is an $S$-full set $W$ in which a superset of $\Lambda$ is distinguished.

Proof By (3.12), $\Lambda$ can be extended to a set $\Gamma$ of wffs which has the following five traits: it is maximal, it is $S$-consistent*, it has the $\square \forall$-property, it also has the $\square^{n}$-property, and it includes $\{@ \mathbf{A}: \mathbf{A} \in \Gamma\}$. Consider the set $P$ of those sets of wffs which have the five traits. By (3.13) $P$ is $S$-full. Let $W$ be the set of just those members $w$ of $P$ such that, for some $n, w$ is, in $P, n$ steps away from $\Gamma$. $W$ is also $S$-full. Moreover, in it $\Gamma$ is distinguished. In particular, for any $w \in W$, if $@ \mathbf{A} \in w$ then $\mathbf{A} \in \Gamma$; for if $\mathbf{A} \notin \Gamma$, then $\neg \mathbf{A} \in \Gamma$, so $@ \neg \mathbf{A} \in w$, in which case, by ST6*, @A $\notin w$.

A model $\left\langle W, R, D, Q, w^{*}, V\right\rangle$ is $S$-canonical just in case it satisfies the following conditions: $W$ is an $S$-full set in which $w^{*}$ is distinguished; for all worlds $w$ and $w^{\prime}$, $w R w^{\prime}$ if and only if $\{\mathbf{A}: \square \mathbf{A} \in w\} \subseteq w^{\prime} ; D$ is the set of variables and individual constants; for every world $w$ and every $\mathbf{b}, w Q \mathbf{b}$ if and only if $w$ contains an atomic wff in which $\mathbf{b}$ occurs; for every $\mathbf{a}, V(\mathbf{a})=\mathbf{a}$; and, for every predicate letter $F_{j}^{i}$, every world $w$, and all $\mathbf{b}_{1}, \ldots, \mathbf{b}_{i}$ that belong to $\operatorname{dom}(w),\left\langle\mathbf{b}_{1}, \ldots, \mathbf{b}_{i}\right\rangle \in V\left(F_{j}^{i}, w\right)$ if and only if $F_{j}^{i} \mathbf{b}_{1} \ldots \mathbf{b}_{i} \in w$. From (3.11) and (3.14) we can derive (3.15).

Metatheorem 3.15 If $\Lambda$ is an S-consistent, finite*, and nonempty actuality set of $w f f$, there is an S-canonical model whose actual world is a superset of $\Lambda$.

We may now prove the following.

Metatheorem 3.16 It is the case in any S-canonical model that, for every $\mathbf{A}$ and every world $w,\left(x_{1}, x_{2}, \ldots\right)$ satisfies $\mathbf{A}$ at $w$ if and only if $\mathbf{A} \in w$.

Proof The proof proceeds, as usual, by induction on the number of occurrences of $\rightarrow, \neg, \square$, @, and $\forall$ in $\mathbf{A}$ and relies on the properties of $S$-full sets. For some details, see [7], pp. 210-11. The only new clause here concerns the case in which $\mathbf{A}$ is @ $\mathbf{B}$; for this case we need to invoke the fact that, in any $S$-canonical model, the actual world is distinguished in the set of worlds.

If $\mathbf{x}_{1}, \ldots, \mathbf{x}_{i}$ are distinct variables arranged in alphabetical order and they are all the variables that have a free occurrence in $\mathbf{A}$, then the closure of $\mathbf{A}, \operatorname{clo}(\mathbf{A})$, will be $\left(\forall \mathbf{x}_{1}\right) \cdots\left(\forall \mathbf{x}_{i}\right) \mathbf{A}$; if no variable has a free occurrence in $\mathbf{A}$, clo(A) will be $\mathbf{A}$ itself. (3.15) and (3.16) imply (3.17).

Metatheorem 3.17 If $\mathbf{A}$ is true in every $S$-canonical model, then $\vdash_{S} \mathbf{A}$.

Proof If $\mathbf{A}$ is true in every $S$-canonical model, so is clo(A). Now assume that $\nvdash_{S} \operatorname{clo}(\mathbf{A})$. Then $\nvdash_{S} @ \operatorname{clo}(\mathbf{A})$. So $\{@ \neg \operatorname{clo}(\mathbf{A})\}$ is $S$-consistent. (For if $\vdash_{S} \neg @ \neg \operatorname{clo}(\mathbf{A})$ then $\vdash_{S} @ \operatorname{clo}(\mathbf{A})$ : we know from $S \mathrm{~T} 4 *$ that there is a proof of 
$\neg @ \neg$ clo(A) $\rightarrow$ @ clo(A) in which @ KA10-11 are not used, so by prefixing such a proof to a proof of $\neg @ \neg \operatorname{clo}(\mathbf{A})$ and then using modus ponens we get a proof of @ clo(A).) Hence, by (3.15), there is an $S$-canonical model whose actual world, $w^{*}$, contains @ $\neg$ clo(A). So, by (3.16), in that model $\left(x_{1}, x_{2}, \ldots\right)$ satisfies @ $\neg$ clo(A) at $w^{*}$. Thus $\left(x_{1}, x_{2}, \ldots\right)$ does not satisfy $\operatorname{clo}(\mathbf{A})$ at $w^{*}$. Let $\mathbf{b} \in \operatorname{dom}\left(w^{*}\right)$. Then by $(3.1)(\mathbf{b}, \mathbf{b}, \ldots)$ does not satisfy $\operatorname{clo}(\mathbf{A})$ at $w^{*}$, contrary to the truth of $\operatorname{clo}(\mathbf{A})$ in every $S$-canonical model. Therefore $\vdash_{S} \operatorname{clo}(\mathbf{A})$, so $\vdash_{S} \mathbf{A}$.

\section{Metatheorem 3.18 If $\mathbf{A}$ is valid in all structures, $\vdash @ \mathrm{~K} \mathbf{A}$.}

(3.18), which states the weak completeness of @ $\mathrm{K}$, is a corollary of (3.17).

Turning to the axiomatic systems @ $j$, we can now easily establish their soundness and weak completeness. The soundness is derived from (3.8). For example, in the case of @1 we should prove that, for every structure meeting condition (1), the instances of the schema @1A are W-true in all models based on that structure. The weak completeness is derived from (3.17). In the case of @ 1, say, we should prove that each@1-canonical model is based on a structure meeting condition (1). As an example, I shall deal with the completeness of @5, @7, @10, @11, and @12.

Metatheorem 3.19 Each @5-canonical model is based on a structure that satisfies condition (5).

Proof We must show that, in each @5-canonical model and for any worlds $w$ and $w^{\prime}$, if $\square \mathbf{A} \in w$ then $\mathbf{A} \in w^{\prime}$. Assume that $\square \mathbf{A} \in w$. Then, by @3A and (3.10), $@ \square \mathbf{A} \in w$, so $\square \mathbf{A}$ belongs to the actual world of the model. Hence, $₫ \square \mathbf{A} \in w^{\prime}$. So, by @1A, $\mathbf{A} \in w^{\prime}$.

Metatheorem 3.20 Each@7-canonical model is based on a structure that satisfies condition (7).

Proof We should first note a general fact: if a maximal $S$-consistent* set $\Gamma$ has the $\forall$-property and contains a wff that can be abbreviated as $(\exists \mathbf{y}) \mathbf{A}$, then there is a variable $\mathbf{x}$ such that $\Gamma$ contains both $\dot{\mathbf{S}}_{\mathbf{x}}^{\mathbf{y}} \mathbf{A}$ and an atomic wff in which $\mathbf{x}$ occurs. For $\Gamma$ does not contain $(\forall \mathbf{y}) \neg[\neg \mathbf{A} \rightarrow \neg \mathbf{A}]$ : if it does, then, contrary to the $S$-consistency* of the set, $(\forall \mathbf{y}) \neg \mathbf{A} \in \Gamma$, since for every $\mathbf{B} \vdash_{S}^{*}(\forall \mathbf{y}) \neg[\mathbf{B} \rightarrow \mathbf{B}] \rightarrow(\forall \mathbf{y}) \mathbf{B}$. Thus, by the $\forall$-property, there is a variable $\mathbf{x}$ and a predicate letter $\mathbf{G}$ such that both $\mathbf{G x}$ and $\dot{\mathbf{S}}_{\mathbf{x}}^{\mathbf{y}} \neg \mathbf{A} \rightarrow(\forall \mathbf{y}) \neg \mathbf{A}$ belong to $\Gamma$. Hence, $\Gamma$ also contains $\neg(\forall \mathbf{y}) \neg \mathbf{A} \rightarrow \dot{\mathbf{S}}_{\mathbf{x}}^{\mathbf{y}} \mathbf{A}$, so $\dot{\mathbf{S}}_{\mathbf{x}}^{\mathbf{y}} \mathbf{A} \in \Gamma$.

Now, we must show that, in each @7-canonical model and for every world $w$, there is a variable or individual constant $\mathbf{b}$ such that $w$ contains an atomic wff in which $\mathbf{b}$ occurs, and the actual world, $w^{*}$, also contains such an atomic wff. We know that, for some $\mathbf{F},\left(\forall x_{1}\right) \mathbf{F} x_{1} \in w^{*}$. Also, by @ 7A, for any $\mathbf{y}$, the wff abbreviated as $(\exists \mathbf{y}) @\left[\left(\forall x_{1}\right) \mathbf{F} x_{1} \rightarrow \mathbf{F y}\right]$ belongs to $w$. So there is a variable $\mathbf{x}$ such that $w$ contains both @ $\left[\left(\forall x_{1}\right) \mathbf{F} x_{1} \rightarrow \mathbf{F x}\right]$ and an atomic wff in which $\mathbf{x}$ occurs. Thus $\left(\forall x_{1}\right) \mathbf{F} x_{1} \rightarrow \mathbf{F x}$ belongs to $w^{*}$, so $\mathbf{F} \mathbf{x} \in w^{*}$.

Metatheorem 3.21 Each @ 10-canonical model is based on a structure that satisfies condition (10).

Proof We must show that, in each @ 10-canonical model and for any worlds $w$ and $w^{\prime}$, if $w$ contains an atomic wff $\mathbf{B}$ in which $\mathbf{b}$ occurs, and $w^{\prime}$ contains an atomic 
wff $\mathbf{C}$ in which $\mathbf{b}$ occurs, then $w$ is $w^{\prime}$. Let $\mathbf{A} \in w$. We know that, for some $n, w^{\prime}$ is $n$ steps away from the actual world, w*. Also, by @10A, w contains $\mathbf{B} \wedge \mathbf{A} \rightarrow @ \square^{n}[\mathbf{C} \rightarrow \mathbf{A}]$. Thus $\square^{n}[\mathbf{C} \rightarrow \mathbf{A}]$ belongs to $w^{*}$, so $\mathbf{A} \in w^{\prime}$. And, conversely, if $\mathbf{A} \in w^{\prime}$, then $\mathbf{A} \in w$. For, if $\mathbf{A} \notin w$, then $\neg \mathbf{A} \in w$, and so $\neg \mathbf{A} \in w^{\prime}$.

Metatheorem 3.22 Each @11-canonical model is based on a structure that satisfies condition (11).

Proof We must show that, in each @ 11-canonical model and for any worlds $w$ and $w^{\prime}$, if $w$ contains an atomic wff $\mathbf{B}$ in which $\mathbf{b}$ occurs, $w^{\prime}$ also contains an atomic wff in which $\mathbf{b}$ occurs. We know that, for some $\mathbf{F}$ and $\mathbf{G},\left(\forall x_{1}\right) \mathbf{F} x_{1} \in w^{*}$ and $\left(\forall x_{1}\right) \mathbf{G} x_{1} \in w^{\prime}$. Also, by @8A, $w$ contains $\mathbf{B} \rightarrow @\left[\left(\forall x_{1}\right) \mathbf{F} x_{1} \rightarrow \mathbf{F b}\right]$. So $\mathbf{F b} \in w^{*}$, and @ $\mathbf{F b} \in w^{\prime}$. But, by @9A, $w^{\prime}$ also contains @ $\mathbf{F b} \rightarrow\left[\left(\forall x_{1}\right) \mathbf{G} x_{1} \rightarrow \mathbf{G b}\right]$. Hence, $\mathbf{G b} \in w^{\prime}$.

Metatheorem $3.23 \quad$ Each @12-canonical model is based on a structure that satisfies condition (12).

Proof We must show that, in each @ 12-canonical model and for any world $w$ such that $\left\{\mathbf{C}: \square \mathbf{C} \in w^{*}\right\} \subseteq w$, if $w^{*}$ (the actual world) contains an atomic wff $\mathbf{B}$ in which $\mathbf{b}$ occurs then $w$ contains no atomic wff in which $\mathbf{b}$ occurs. Suppose that such a wff $\mathbf{A}$ belongs to $w$, let $\mathbf{x}$ be a variable that does not occur in $\mathbf{A}$, and let $\mathbf{A}^{\prime}$ result from $\mathbf{A}$ by replacing $\mathbf{b}$ with $\mathbf{x}$. Then, by @ 12A, $w^{*}$ contains $(\forall \mathbf{x}) \square \neg \mathbf{A}^{\prime}$, and by @KA7 it contains $\mathbf{B} \rightarrow\left[(\forall \mathbf{x}) \square \neg \mathbf{A}^{\prime} \rightarrow \square \neg \mathbf{A}\right]$. Thus $\neg \mathbf{A} \in w$, contrary to the @ 12-consistency* of $w$.

Now, in order to demonstrate compactness and strong completeness, we extend the language $\mathcal{L}$ to a language $\mathcal{L}^{+}$by adding the denumerably many variables $y_{1}, y_{2}, \ldots$ and the denumerably many predicate letters $G_{1}^{1}, G_{2}^{1}, \ldots$ and $H_{1}^{1}, H_{2}^{1}, \ldots$ of degree 1. The variables of $\mathscr{L}^{+}$, old and new, are enumerated. The concept of a model ${ }^{+}$is defined in the context of $\mathcal{L}^{+}$just as the concept of a model was defined in the context of $\mathcal{L}$. Given a model ${ }^{+}\left\langle W, R, D, Q, w^{*}, V^{+}\right\rangle$and a sequence $s$ of elements of $D$, the function $s^{+}$is the following: for every variable $\mathbf{v}$ of $\mathscr{L}^{+}, s^{+}(\mathbf{v})=s_{k}$ where $\mathbf{v}$ is the $k$ th variable in the enumeration of the variables of $\mathcal{L}^{+} ; s^{+}(\mathbf{a})=V^{+}(\mathbf{a})$. Satisfaction in a model ${ }^{+}$is defined like satisfaction in a model, except that now the basic clause relies on the function $s^{+}$, and the clause for $\forall$ relies on the enumeration of all the variables of $\mathcal{L}^{+}$. The axiomatic system @ $\mathrm{K}^{+}$will be just like @ $\mathrm{K}$ except that it concerns the language $\mathcal{L}^{+}$. Other model-theoretic and proof-theoretic concepts are also extended to the new language. I assume that we have proved the analogues in $\mathcal{L}^{+}$of various metatheorems we proved above for $\mathcal{L}$.

Metatheorem 3.24 For any model $M=\left\langle W, R, D, Q, w^{*}, V\right\rangle$ and any mode $^{+} M^{+}=\left\langle W, R, D, Q, w^{*}, V^{+}\right\rangle$where $V \subset V^{+}$, for any wff $\mathbf{A}$ of $L$, for any $w \in W$, and for any sequences $s$ and $s^{\prime}$ of elements of $D$ such that, for every $i$, $s^{*}\left(x_{i}\right)=s^{\prime+}\left(x_{i}\right), s$ satisfies $\mathbf{A}$ at $w$ in $M$ if and only if $s^{\prime}$ satisfies $\mathbf{A}$ at $w$ in $M^{+}$.

(3.24) can be proved by induction on the number of occurrences of $\rightarrow, \neg, \square$, @, and $\forall$ in $\mathbf{A}$.

We shall now establish that satisfiability in a structure is compact. 
Metatheorem 3.25 Let $\Lambda$ be any set of wffs of $\mathcal{L}$. If, for each finite subset of $\Lambda$, there is a structure in which that subset is satisfiable, then $\Lambda$ is satisfiable in a structure.

Proof We assume that $\Lambda$ is not finite, for the case of finite $\Lambda$ is trivial. Let $\Delta$ be the set of wffs of $\mathcal{L}$ which results from $\Lambda$ when we prefix @ to each wff in it, and let $\Theta$ be $\Delta \cup\left\{@ G_{1}^{1} x_{1}, @ G_{2}^{1} x_{2}, \ldots\right\}$.

Consider any finite subset $\Delta^{\prime}$ of $\Delta$. We can see that there is a model $M=$ $\left\langle W, R, D, Q, w^{*}, V\right\rangle$ in which, for some sequence $s$ of elements of $\operatorname{dom}\left(w^{*}\right)$, $s$ satisfies all members of $\Delta^{\prime}$ at $w^{*}$. Further, we can construct a model ${ }^{+} M^{+}=$ $\left\langle W, R, D, Q, w^{*}, V^{+}\right\rangle$where $V^{+} \supset V$ and, for every $i, V^{+}\left(G_{i}^{1}, w^{*}\right)=\operatorname{dom}\left(w^{*}\right)$. Finally, let $s^{\prime}$ be a sequence of elements of $\operatorname{dom}\left(w^{*}\right)$ such that, for every $i$, $s^{\prime+}\left(x_{i}\right)=s^{*}\left(x_{i}\right)$. Then (3.24) implies that, in $M^{+}, s^{\prime}$ satisfies all members of $\Delta^{\prime}$ at $w^{*}$. But, in $M^{+}, s^{\prime}$ also satisfies all members of $\left\{@ G_{1}^{1} x_{1}, @ G_{2}^{1} x_{2}, \ldots\right\}$ at $w^{*}$. In other words, $\Delta^{\prime} \cup\left\{@ G_{1}^{1} x_{1}, @ G_{2}^{1} x_{2}, \ldots\right\}$ is satisfiable in a structure. Hence, so is every finite subset of $\Theta$.

Suppose that $\Theta$ is not $@ \mathrm{~K}^{+}$-consistent. Then there will be wffs $\mathbf{A}_{1}^{+}, \ldots, \mathbf{A}_{j}^{+}$of $\mathcal{L}^{+}$such that $\mathbf{A}_{1}^{+}, \ldots, \mathbf{A}_{j}^{+} \in \Theta$ and $\vdash_{@ \mathrm{~K}^{+}} \neg\left[\mathbf{A}_{1}^{+} \wedge \cdots \wedge \mathbf{A}_{j}^{+}\right]$. So, by the soundness of $@ \mathrm{~K}^{+}, \neg\left[\mathbf{A}_{1}^{+} \wedge \cdots \wedge \mathbf{A}_{j}^{+}\right]$will be valid in every structure, contrary to the satisfiability of $\left\{\mathbf{A}_{1}^{+}, \ldots, \mathbf{A}_{j}^{+}\right\}$in some structure. Hence $\Theta$ is $@ \mathrm{~K}^{+}$-consistent.

$\Theta$ is a nonempty actuality set, and it is finite* in $\mathcal{L}^{+}$. Let $s^{\prime}$ now be the sequence of all the variables of $\mathcal{L}^{+}$in increasing order of their enumeration. By the analogues of (3.15) and (3.16) for $\mathcal{L}^{+}$, there is an $@ \mathrm{~K}^{+}$-canonical model ${ }^{+} M^{+}=$ $\left\langle W, R, D, Q, w^{*}, V^{+}\right\rangle$in which $s^{\prime}$ satisfies every member of $\Theta$ at $w^{*}$. Thus, in $M^{+}$, $s^{\prime}$ satisfies all of $G_{1}^{1} x_{1}, G_{2}^{1} x_{2}, \ldots$ at $w^{*}$. So, for every $i, s^{\prime+}\left(x_{i}\right)$, which is $x_{i}$ itself, belongs to $\operatorname{dom}\left(w^{*}\right)$.

Consider the model $M$ that results from $M^{+}$when we delete the valuation of the predicate letters that extend $\mathcal{L}$ to $\mathcal{L}^{+}$. (3.24) shows that, in $M,\left(x_{1}, x_{2}, \ldots\right)$ satisfies all members of $\Delta$ at $w^{*}$, and thus it satisfies all members of $\Lambda$ at $w^{*}$.

Proceeding as in the proof of (3.25) and relying on metatheorems for $\mathcal{L}^{+}$such as (3.19) - (3.23), we can also demonstrate, for every $j(1 \leq j \leq 12)$, that satisfiability in a structure that meets condition $(j)$ is compact. In other words, if, for each finite subset of $\Lambda$, there is a structure which meets $(j)$ and in which that subset is satisfiable, then $\Lambda$ itself is satisfiable in a structure that meets $(j)$.

It is time to prove the strong completeness of @ $\mathrm{K}$.

Metatheorem 3.26 Let $\mathbf{A}$ be a wff of $\mathcal{L}$, and $\Lambda$ be a set of wffs of $\mathcal{L}$. If $\mathbf{A}$ is a consequence of $\Lambda$ in every structure, then for some subset $\left\{\mathbf{B}_{1}, \ldots, \mathbf{B}_{n}\right\}$ of $\Lambda(n \geq 0)$ $\vdash_{@ \mathrm{~K}} \mathbf{B}_{1} \wedge \cdots \wedge \mathbf{B}_{n} \rightarrow \mathbf{A}$.

Proof Since $\mathbf{A}$ is a consequence of $\Lambda$ in every structure, there is no structure in which $\Lambda \cup\{\neg \mathbf{A}\}$ is satisfiable. Thus, by (3.25), for some subset $\left\{\mathbf{B}_{1}, \ldots, \mathbf{B}_{n}\right\}$ of $\Lambda$ there is no structure in which $\left\{\mathbf{B}_{1}, \ldots, \mathbf{B}_{n}, \neg \mathbf{A}\right\}$ is satisfiable. So $\mathbf{B}_{1} \wedge \cdots \wedge \mathbf{B}_{n} \rightarrow \mathbf{A}$ is valid in every structure. We can then invoke the weak completeness of @ $\mathrm{K}$.

Proceeding as in the proof of (3.26), we can prove the strong completeness of @ $j$, for $1 \leq j \leq 12$. In other words, if $\mathbf{A}$ is a consequence of $\Lambda$ in every structure that meets condition $(j)$, then for some subset $\left\{\mathbf{B}_{1}, \ldots, \mathbf{B}_{n}\right\}$ of $\Lambda(n \geq 0)$ $\vdash_{@ j} \mathbf{B}_{1} \wedge \cdots \wedge \mathbf{B}_{n} \rightarrow \mathbf{A}$. 
Now, in order to see why the theorems of @K (or those of @ $j$ ) which belong to the language $\mathcal{L}^{\prime}$ (the nonmodal part of $\mathcal{L}$ ) are just the wffs that make up the classical predicate calculus without identity when this calculus is formulated in $\mathcal{L}^{\prime}$, we may follow the reasoning deployed in [7], pp. 213-14. Note that, in establishing the analogue of Metatheorem 3.19 of that paper, we must use both ST10 and ST18 (of the present paper).

We should turn to the axiomatizations that introduce a sign of identity. Our first task is to prove analogues of (3.6) - (3.8). For any set $\Lambda$ of axiom schemata, @IK + $\Lambda$ will be the axiomatic system that results from @IK by adding those schemata.

Metatheorem 3.27 If, for any structure, $@ \square^{n}(\forall \mathbf{x}) \mathbf{F x} \rightarrow \mathbf{A}$, where $\mathbf{F}$ does not occur in $\mathbf{A}$, is $W$-true in every identity model based on that structure, then $\mathbf{A}$ is also $W$-true in every identity model based on that structure.

Metatheorem 3.28 If, for any structure, $(\forall \mathbf{x}) \mathbf{F x} \rightarrow \mathbf{A}$, where $\mathbf{F}$ does not occur in $\mathbf{A}$, is $W$-true in every identity model based on that structure, then $\mathbf{A}$ is also $W$-true in every identity model based on that structure.

Metatheorem 3.29 If, for any structure, $\Lambda$ is a set of axiom schemata whose instances are $W$-true in every identity model based on that structure, the theorems of $@ \mathrm{IK}+\Lambda$ are I-valid in the structure.

The proofs of (3.27) and (3.28) are similar to those of (3.6) and (3.7). The proof of (3.29) is similar to that of (3.8) but makes use of (3.27) and (3.28) and of the fact that the instances of the schemata @IKA1-3 are W-true in every identity model.

Relying on (3.29), we can easily establish the soundness of @IK and @ $\mathrm{I} j$ $(1 \leq j \leq 12)$. In order to demonstrate their completeness, we must prove some theorems in those systems. Let $S I$ be an arbitrary axiomatic system which differs from @IK in at most having additional axiom schemata such that if $\mathbf{A}$ is an instance of one of those schemata, $\mathbf{a}_{1}, \ldots, \mathbf{a}_{n}$ are distinct individual constants, and $\mathbf{y}_{1}, \ldots, \mathbf{y}_{n}$ are variables that do not occur in $\mathbf{A}$, then $\mathbf{S}_{\mathbf{y}_{1}, \ldots, \mathbf{y}_{n}}^{\mathbf{a}_{1}, \ldots, \mathbf{a}_{n}} \mathbf{A}$ is also an instance of the relevant schema. Any such axiomatic system is among those designated by $S$, so we can speak of $S I$-fullness, $S I$-consistency, and so forth, on the basis of definitions that have already been given. For a proof of $S I T 2^{*}, S I T 3^{*}$, and SIT4*, see [7], pp. 214-15.

SIT1* $\quad \mathbf{A} \rightarrow \mathbf{b}=\mathbf{b}$ where $\mathbf{A}$ is an atomic wff in which $\mathbf{b}$ occurs.

Proof Let $\mathbf{x}$ be any variable. Then we need @IKA1 and $\mathbf{A} \rightarrow[(\forall \mathbf{x})[\mathbf{x}=\mathbf{x}]$ $\rightarrow \mathbf{b}=\mathbf{b}]$, which is an instance of @ KA7.

$$
\begin{array}{ll}
S I T 2^{*} & \mathbf{b}=\mathbf{c} \rightarrow \mathbf{c}=\mathbf{b} . \\
S I \mathrm{~T} 3^{*} & \mathbf{b}=\mathbf{c} \rightarrow[\mathbf{c}=\mathbf{d} \rightarrow \mathbf{b}=\mathbf{d}] . \\
{S I T 4^{*}} & \mathbf{b}_{1}=\mathbf{c}_{1} \rightarrow\left[\cdots \rightarrow\left[\mathbf{b}_{i}=\mathbf{c}_{i} \rightarrow\left[\mathbf{F b}_{1} \ldots \mathbf{b}_{i} \rightarrow \mathbf{F c}_{1} \ldots \mathbf{c}_{i}\right]\right] \ldots\right] . \\
{S I T 5^{*}} & \mathbf{b}=\mathbf{c} \rightarrow @ \square^{n}[\mathbf{A} \rightarrow \mathbf{b}=\mathbf{c}] \text { where } \mathbf{A} \text { is an atomic wff in which } \mathbf{b} \\
& \text { occurs or } \mathbf{c} \text { occurs. }
\end{array}
$$

Proof Let $\mathbf{x}$ and $\mathbf{y}$ be distinct variables that do not occur in $\mathbf{b}=\mathbf{c} \rightarrow @^{n}[\mathbf{A}$ $\rightarrow \mathbf{b}=\mathbf{c}$ ]. Let $\mathbf{A}^{\prime}$ result from $\mathbf{A}$ by substituting $\mathbf{y}$ for every occurrence of $\mathbf{c}$, and let $\mathbf{A}^{\prime \prime}$ result from $\mathbf{A}^{\prime}$ by substituting $\mathbf{x}$ for every occurrence of $\mathbf{b}$. Note that if $\mathbf{b}$ and $\mathbf{c}$ are the same symbol, then $\mathbf{A}^{\prime \prime}$ is $\mathbf{A}^{\prime}$. Also, $\mathbf{x}$ does not occur in $\mathbf{A}^{\prime} . \quad \mathbf{x}=\mathbf{y} \rightarrow @ \square^{n}\left[\mathbf{A}^{\prime \prime} \rightarrow \mathbf{x}=\mathbf{y}\right]$ is an instance of @IKA3. Hence 
$(\forall \mathbf{x})(\forall \mathbf{y})\left[\mathbf{x}=\mathbf{y} \rightarrow \square^{n}\left[\mathbf{A}^{\prime \prime} \rightarrow \mathbf{x}=\mathbf{y}\right]\right]$. Thus, by @KA7, $\mathbf{b}=\mathbf{c} \rightarrow(\forall \mathbf{y})[\mathbf{b}=\mathbf{y}$ $\left.\rightarrow @ \square^{n}\left[\mathbf{A}^{\prime} \rightarrow \mathbf{b}=\mathbf{y}\right]\right]$. But by $@ \mathrm{KA} 7$ we also have $\mathbf{b}=\mathbf{c} \rightarrow[(\forall \mathbf{y})[\mathbf{b}=\mathbf{y}$ $\left.\left.\rightarrow @ \square^{n}\left[\mathbf{A}^{\prime} \rightarrow \mathbf{b}=\mathbf{y}\right]\right] \rightarrow\left[\mathbf{b}=\mathbf{c} \rightarrow @ \square^{n}[\mathbf{A} \rightarrow \mathbf{b}=\mathbf{c}]\right]\right]$

The standard order of variables and individual constants will be $x_{1}, a_{1}, x_{2}, a_{2}, \ldots$. Given an $S I$-full set $W$, we can define the function $f$ from variables and individual constants: for each $\mathbf{b}, f(\mathbf{b})$ is the first individual constant or variable $\mathbf{c}$ in the standard order such that, for every world $w \in W$ containing an atomic wff in which $\mathbf{b}$ or $\mathbf{c}$ occurs, $\mathbf{b}=\mathbf{c} \in w .^{8}$ It is a consequence of $S I \mathrm{~T} 1^{*}$ that, for every world $w \in W$ containing an atomic wff in which $\mathbf{b}$ occurs, $\mathbf{b}=\mathbf{b} \in w$.

A model $\left\langle W, R, D, Q, w^{*}, V\right\rangle$ is $S I$-identity-canonical just in case it is an identity model and satisfies the following conditions: $W$ is an $S I$-full set in which $w^{*}$ is distinguished; for all worlds $w$ and $w^{\prime}, w R w^{\prime}$ if and only if $\{\mathbf{A}: \square \mathbf{A} \in w\} \subseteq w^{\prime} ; D$ is the range of $f$; for every world $w$ and every $\mathbf{b} \in D, w Q \mathbf{b}$ if and only if $w$ contains an atomic wff in which $\mathbf{b}$ occurs; for every $\mathbf{a}, V(\mathbf{a})$ is $f(\mathbf{a})$; and, for every predicate letter $F_{j}^{i}$ other than $F_{1}^{2}$, for every world $w$, and for all $\mathbf{b}_{1}, \ldots, \mathbf{b}_{i}$ that belong to $\operatorname{dom}(w),\left\langle\mathbf{b}_{1}, \ldots, \mathbf{b}_{i}\right\rangle \in V\left(F_{j}^{i}, w\right)$ if and only if $F_{j}^{i} \mathbf{b}_{1} \ldots \mathbf{b}_{i} \in w$. From (3.11) and (3.14) we can derive (3.30).

Metatheorem 3.30 If $\Lambda$ is an SI-consistent, finite*, and nonempty actuality set of wffs, there is an SI-identity-canonical model whose actual world is a superset of $\Lambda .{ }^{9}$

The following is analogous to (3.16).

Metatheorem 3.31 It is the case in any SI-identity-canonical model that, for every $\mathbf{A}$ and every world $w,\left(f\left(x_{1}\right), f\left(x_{2}\right), \ldots\right)$ satisfies $\mathbf{A}$ at $w$ if and only if $\mathbf{A} \in w$.

Proof We shall only discuss the case in which $\mathbf{A}$ is $F_{1}^{2} \mathbf{b}_{1} \mathbf{b}_{2}$, and so can be abbreviated as $\mathbf{b}_{1}=\mathbf{b}_{2}$, while $f\left(\mathbf{b}_{1}\right), f\left(\mathbf{b}_{2}\right) \in \operatorname{dom}(w)$. Then $w$ contains $\mathbf{b}_{1}=f\left(\mathbf{b}_{1}\right)$ and $\mathbf{b}_{2}=f\left(\mathbf{b}_{2}\right)$. Also $\left(f\left(x_{1}\right), f\left(x_{2}\right), \ldots\right)$ satisfies $\mathbf{A}$ at $w$ if and only if $\left\langle f\left(\mathbf{b}_{1}\right), f\left(\mathbf{b}_{2}\right)\right\rangle \in V\left(F_{1}^{2}, w\right)$ if and only if $f\left(\mathbf{b}_{1}\right)$ is $f\left(\mathbf{b}_{2}\right)$. Now, if $f\left(\mathbf{b}_{1}\right)$ is $f\left(\mathbf{b}_{2}\right)$, then $\mathbf{b}_{1}=f\left(\mathbf{b}_{2}\right) \in w$; but by SIT2* $f\left(\mathbf{b}_{2}\right)=\mathbf{b}_{2} \in w$; thus, by SIT3*, $\mathbf{b}_{1}=\mathbf{b}_{2} \in w$. On the other hand, if $f\left(\mathbf{b}_{1}\right)$ is other than $f\left(\mathbf{b}_{2}\right)$, let $f\left(\mathbf{b}_{1}\right)$ appear earlier than $f\left(\mathbf{b}_{2}\right)$ in the standard order. Then there is a world $w^{\prime}$ which does not contain $\mathbf{b}_{2}=f\left(\mathbf{b}_{1}\right)$ but contains an atomic wff $\mathbf{C}$ in which $\mathbf{b}_{2}$ or $f\left(\mathbf{b}_{1}\right)$ occurs. For some $n, w^{\prime}$ is $n$ steps away from $w^{*}$. Assume that $\mathbf{b}_{2}=f\left(\mathbf{b}_{1}\right)$ belongs to $w$. Then, by SIT5*, $w$ also contains $@ \square^{n}\left[\mathbf{C} \rightarrow \mathbf{b}_{2}=f\left(\mathbf{b}_{1}\right)\right]$, and so $w^{*}$ contains $\square^{n}\left[\mathbf{C} \rightarrow \mathbf{b}_{2}=f\left(\mathbf{b}_{1}\right)\right]$. But then $\mathbf{b}_{2}=f\left(\mathbf{b}_{1}\right)$ belongs to $w^{\prime}$, contrary to what we know. Hence $\mathbf{b}_{2}=f\left(\mathbf{b}_{1}\right)$ does not belong to $w$. Therefore, since $\mathbf{b}_{1}=f\left(\mathbf{b}_{1}\right) \in w$, $w$ does not contain $\mathbf{b}_{2}=\mathbf{b}_{1}$, and so it does not contain $\mathbf{b}_{1}=\mathbf{b}_{2}$. Things are similar if $f\left(\mathbf{b}_{2}\right)$ appears earlier than $f\left(\mathbf{b}_{1}\right)$ in the standard order.

For the case in which $\mathbf{A}$ is @ $\mathbf{B}$, see the proof of (3.16). For other cases, see [7], pp. $215-16$.

(3.30) and (3.31) imply the next metatheorem.

Metatheorem 3.32 If $\mathbf{A}$ is true in every SI-identity-canonical model, then $\vdash_{S I} \mathbf{A}$.

The proof of (3.32) is like that of (3.17). Using (3.32), we can demonstrate the weak completeness of the axiomatic systems@ @IK and @I $j(1 \leq j \leq 12)$ : the proof is essentially the same as the proof of weak completeness for their counterparts without identity, except that a few additional steps are needed in the reasoning for 
@ I7. For example, the wffs that are I-valid in every structure meeting condition (1) are theorems of @I1.

Proceeding as in the proofs of (3.25) and (3.26), we can establish compactness of I-satisfiability, as well as strong completeness, for the systems @ IK and @ $\mathrm{I} j$. As regards @I1, for example, it is the case for any set $\Lambda$ of wffs that if, for each finite subset of $\Lambda$, there is a structure which meets condition (1) and in which that subset is I-satisfiable, then $\Lambda$ itself is I-satisfiable in a structure meeting (1); and if $\mathbf{A}$ is an I-consequence of $\Lambda$ in every structure that meets (1), then for some subset $\left\{\mathbf{B}_{1}, \ldots, \mathbf{B}_{n}\right\}$ of $\Lambda(n \geq 0) \vdash @ \mathrm{II} \mathbf{B}_{1} \wedge \cdots \wedge \mathbf{B}_{n} \rightarrow \mathbf{A}$.

Finally, in order to see why the theorems of @IK (or those of @ $\mathrm{I} j$ ) which belong to the language $\mathcal{L}^{\prime}$ are just the wffs that make up the classical predicate calculus with identity when this calculus is formulated in $\mathscr{L}^{\prime}$ and the sign of identity is $F_{1}^{2}$, we may follow the reasoning deployed in [7], pp. 216-17.

\section{Notes}

1. I happen to believe that an operator which always introduces information about what is in fact the case captures only some of the aspects of the notion that is expressed in natural languages in words like 'actually' and its cognates. Be that as it may, it is still the case that adding such an operator to the usual idiom of modal logic goes a long way toward rectifying the neglect of actuality.

2. There is an exception to this generalization. In the proof of (3.31) below, the case in which $\mathbf{A}$ can be abbreviated as $\mathbf{b}_{1}=\mathbf{b}_{2}$ is treated in a way that involves the symbol @ essentially and so could not have been followed in the other paper.

3. A statement such as ' $\mathbf{A} \vee \neg \mathbf{A}$ is a theorem' will mean 'The wff abbreviated as $\mathbf{A} \vee \neg \mathbf{A}$ is a theorem'.

4. Outermost brackets are omitted. The rule of the association to the left applies; for example, $\mathbf{A} \rightarrow \mathbf{B} \rightarrow \mathbf{C}$ abbreviates $[[\mathbf{A} \rightarrow \mathbf{B}] \rightarrow \mathbf{C}$ ]. The brackets that enclose a disjunction or conjunction are omitted when the disjunction or conjunction is an argument of an occurrence of $\rightarrow$ or $\leftrightarrow$; so $\mathbf{A} \rightarrow \mathbf{B} \vee \mathbf{C}$ abbreviates $[\mathbf{A} \rightarrow[\mathbf{B} \vee \mathbf{C}]]$. No other brackets are omitted.

5. Given a structure, a world $w^{\prime}$ is $0 R$-steps away from $w$ if and only if it is $w$ itself, and it is $m+1 R$-steps away from $w$ if and only if there is a world $w^{\prime \prime}$ that is $m R$-steps away from $w$ while $w^{\prime \prime} R w^{\prime}$.

6. ST12*, as well as (3.3), should be used in the proofs of (3.16) and (3.31) below.

7. The requirement that each set in $W$ should contain a wff of the form $\left(\forall x_{1}\right) \mathbf{F} x_{1}$ contributes to proving the completeness of @7, @8, @9, @11, and the corresponding systems with identity.

8. A statement such as ' $\mathbf{b}=\mathbf{c}$ belongs to $w$ ' will mean 'The wff abbreviated as $\mathbf{b}=\mathbf{c}$ belongs to $w$ '. 
9. In order to derive (3.30), note that if a member $\Gamma$ of an $S I$-full set contains an atomic wff in which a constant a occurs, then $\Gamma$ also contains an atomic wff in which $f(\mathbf{a})$ occurs e.g., the wff $F_{1}^{2} \mathbf{a} f(\mathbf{a})$.

\section{References}

[1] Blackburn, P., and M. Marx, "Remarks on Gregory's 'actually' operator," Journal of Philosophical Logic, vol. 31 (2002), pp. 281-88. Zbl 1003.03016. MR 1917268. 382

[2] Crossley, J. N., and L. Humberstone, "The logic of 'actually'," Reports on Mathematical Logic, vol. 8 (1977), pp. 11-29. Zbl 0389.03010. MR 0485193. 382

[3] Gregory, D., "Completeness and decidability results for some propositional modal logics containing 'actually' operators," Journal of Philosophical Logic, vol. 30 (2001), pp. 57-78. Zbl 0988.03035. MR 1829608. 382

[4] Hodes, H. T., “Axioms for actuality,” Journal of Philosophical Logic, vol. 13 (1984), pp. 27-34. Zbl 0538.03015. MR 737516. 382

[5] Hughes, G. E., and M. J. Cresswell, A New Introduction to Modal Logic, Routledge, London, 1996. Zbl 0855.03002. MR 1666445. 393

[6] Plantinga, A., "Replies to my colleagues," pp. 313-96 in Alvin Plantinga, edited by J. E. Tomberlin and P. van Inwagen, D. Reidel Publishing Co., Dordrecht, 1985. 382

[7] Stephanou, Y., "Investigations into quantified modal logic," Notre Dame Journal of Formal Logic, vol. 43 (2002), pp. 193-220. Zbl 02104804. MR 2034746. 382, 391, 393, 398, 402, 403, 404

\section{Acknowledgments}

This paper was written mostly while I was holding a British Academy Postdoctoral Fellowship. I am also indebted to the anonymous referee for the NDJFL, whose comments led to some improvements.

Department of Philosophy and History of Science

University of Athens

Ilissia Campus

15771 Athens

GREECE

ystephan@phs.uoa.gr 